\title{
Evolution of Multiphase Hot Interstellar Medium in Elliptical Galaxies
}

\author{
Yutaka Fujita円 \\ Graduate School of Human and Environmental Studies, \\ Kyoto University, Kyoto 606-01, Japan \\ Yukawa Institute for Theoretical Physics, \\ Kyoto University, Kyoto 606-01, Japan \\ Junji Fukumoto \\ Nihon Silicon Graphics Cray K.K., Cuore Bldg., 9th Floor, 12-25, \\ Hiroshiba-cho, Suita-si, Osaka 564, Japan \\ Katsuya Okoshi \\ Department of Earth and Space Science,Faculty of Science, \\ Osaka University,Machikaneyama-cho, Toyonaka, Osaka 560, Japan
}

To appear in The Astrophysical Journal

\begin{abstract}
We present the results of a variety of simulations concerning the evolution of multiphase (inhomogeneous) hot interstellar medium (ISM) in elliptical galaxies. We assume the gases ejected from stars do not mix globally with the circumferential gas. The ejected gas components evolve separately according to their birth time, position, and origin. We consider cases where supernova remnants (SNRs) mix with local ISM. The components with high metal abundance and/or high density cool and drop out of the hot ISM gas faster than the other components because of their high metal abundance and/or density. This makes the average metal abundance of the hot ISM low. Furthermore, since the metal abundance of mass-loss gas decreases with radius, gas inflow from outer region makes the average metal abundance of the hot ISM smaller than that of mass-loss gas in the inner region. As gas ejection rate of stellar system decreases, mass fraction of mass-loss gas ejected at outer region increases in a galaxy. If the mixing of SNRs is ineffective, our model predicts that observed $[\mathrm{Si} / \mathrm{Fe}]$ and $[\mathrm{Mg} / \mathrm{Fe}]$ should decrease towards the galactic center because of strong iron emission by SNRs. In the outer region, where the cooling of time of the ISM is long, the selective cooling is ineffective and most of gas components remain hot. Thus, the metal abundance of the ISM in this region directly reflects that of the gas ejected from
\end{abstract}

\footnotetext{
${ }^{1}$ Present Address : Department of Physics, Tokyo Metropolitan University, Minami-Ohsawa 1-1, Hachioji, Tokyo 192-03, Japan : yfujita@phys.metro-u.ac.jp
} 
stars. Our model shows that supernovae are not effective heating sources in the inner region of elliptical galaxies, because most of the energy released by them radiates. Therefore, cooling flow is established even if the supernova rate is high. Mixing of SNRs with ambient ISM makes the energy transfer between supernova explosion and ambient ISM more effective.

Subject headings: galaxies : intergalactic medium - galaxies : interstellar matter galaxies : X-rays

\section{INTRODUCTION}

Observations by X-ray satellites have shown that the X-ray properties of bright elliptical galaxies can be explained by thermal emission of hot interstellar medium (ISM). The estimated

temperatures are around $1 \mathrm{keV}$ and X-ray luminosities are typically around $10^{40} \mathrm{erg}$. Theoretical arguments indicate that the ISM is inhomogeneous; Mathews (1990) estimated that the $\sim 1 M_{\odot}$ of metal ejected by each supernova event into the ISM is trapped locally within the hot bubbles. Since in elliptical galaxies, there is no overlapping of expanding supernova remnants after galactic wind period (Fujita, Fukumoto, \& Okoshi 1996 ; hereafter Paper I), it is expected that this inhomogeneity persists for a long time. The recent observation of ROSAT supports this idea by showing existence of components with several temperatures in elliptical galaxies. (Kim \& Fabbiano 1995)

Based on these arguments, we studied the evolution of the multiphase (inhomogeneous) ISM in Paper I. We showed that the gas components with high metal abundance and high density cool and drop out of the hot ISM faster than other components. As gas ejection rate of stellar system decreases, gas components with low metal abundance dominate in a galaxy. As a result, the average metal abundance which we predict is lower than that predicted by the previous homogeneous ISM models (e.g. Arimoto \& Yoshii 1987 ; Matteucci \& Tornambé 1987 ; Loewenstein \& Mathews 1991 ; Renzini et al. 1993 ; Mihara \& Takahara 1994 ; Matteucci \& Gibson 1995 ; Fukumoto \& Ikeuchi 1996). The low metal abundance is consistent with the observations. (Awaki et al. 1994 ; Loewenstein et al. 1994 ; Matsushita et al. 1994 ; Mushotzky et al. 1994 ; Kim \& Fabbiano 1995 ; Davis \& White 1996 ; Matsumoto et al. 1996). We also predicted in Paper I that the heating by supernovae is not effective because the gas components originated from supernovae remnants have high metal abundance, and their energy radiates before transfered to the circumferential ISM.

However, since our model was a simple one-zone model, it could not predict spatial variation in the ISM, which is recently observed by ASCA (Mushotzky et al. 1994) and ROSAT (Kim \& Fabbiano 1995 ; Rangarajan et al. 1995 ; Irwin \& Sarazin 1996). In this paper, we investigate 
the radial evolution of the ISM assuming that the gases ejected from stars do not mix with the ambient ISM globally.

The paper is organized as follows. In $\S 2$ we describe our method for simulating the evolution of the multiphase hot ISM in elliptical galaxies. Then, in $\S 3$, we solve these equations numerically for some typical models. Our results are summarized in $\S 4$.

\section{ASSUMPTION AND BASIC EQUATIONS}

We consider the evolution of the hot ISM after galactic wind period. We assume for simplicity that an elliptical galaxy is spherically symmetric. The model galaxies we used here are the same as those of Loewenstein \& Mathews (1987). The stellar density distribution is

$$
\rho_{\star}(R)=\rho_{0 \star}\left[1+\left(R / R_{a \star}\right)^{2}\right]^{-3 / 2}
$$

for $R<R_{t}$, where $R$ is the distance form the center of the galaxy and $R_{t}$ is the tidal radius. The density distribution of dark halo is

$$
\rho_{h}(R)=\rho_{0 h}\left[1+\left(R / R_{a h}\right)^{2}\right]^{-1}
$$

for $R<R_{t}$. The subscripts $a$ and 0 refer to core and central properties. Both densities are assumed to vanish for $R>R_{t}$. The properties of the model galaxies are shown in Table 1 . The stellar velocity dispersion profile, $\sigma_{\star}(R)$, is derived by equation (23) of Sarazin \& White (1987), which is valid for an isotropic velocity dispersion.

The ISM of the galaxy is divided into finite number zones, $0<R_{1}(t)<\ldots<R_{j}(t)<\ldots<$ $R_{m}(t)$, Each zone moves with the ISM. The zone, $R_{j-1}<R<R_{j}$, is called the $j$ th zone. When $R_{j-1}>R_{t}$, the $j$ th zone is deleted. Furthermore, when $R_{j}<0.12 \mathrm{kpc}$, the $j$ th zone is also deleted to avoid too many time-steps. Each zone is assumed to be uniform on average, although it contains many gas components (hereafter called "phases"). For simplicity, we assume that the phases do not globally mix with ambient gas and comove with the zones which they belong to. These assumptions are valid as long as magnetic field suppresses relative motion of the phases (Hattori, Yoshida, \& Habe 1995). We will discuss the validity in $\$ 2.1 .3$. The phases are classified by their origin, birth time, and zone which they belong to. We consider the three types of the origin, that is, gas ejected through stellar wind (mass-loss gas), shell and cavity of supernova remnants (SNRs). The phases originated from mass-loss gas are called "mass-loss phases" indicated by the index $M L$. Since the cavity of a SNR is buoyant, it floats and breaks part of the shell, and mixes with the ambient ISM locally. These partially broken shell and mixed cavity evolve as separate phases. They are called "bored shell phases (BSPs)" and "mixed cavity phases (MCPs)" indicated by the indices $b s$ and $m c$, respectively. We divide time into a finite number of steps for the $j$ th zone, $0<t_{0, j}<t_{1, j}<\ldots<t_{i, j}<\ldots<t_{n, j}$ (see equation(56)), where $t_{0, j}\left(=t_{0}\right)$ and $t_{n, j}\left(=t_{f}\right)$ are 
the start and end time of the calculation, respectively. The both times are independent of the zones. We define $t=t_{0}(=0.5 \mathrm{Gyr})$ as the time when the galactic wind stops. In each time-step, one phase is born for each type. The phase of type $\alpha(M L, b s$, or $m c)$ which is born in $t_{i-1, j}<t<t_{i, j}$ and in the $j$ th zone is called the $(i, j, \alpha)$-th phase and has its own temperature $T^{(i, j, \alpha)}$, density $\rho^{(i, j, \alpha)}$, metal abundance $Z^{(i, j, \alpha)}$, and mass $M^{(i, j, \alpha)}$. Each phase radiates its thermal energy and evolves. We assume that energy transfer between the phases is worked by pressure. Therefore, the phases are not independent each other. We ignore thermal conduction for simplicity. The phases are assumed to be in pressure equilibrium because sound crossing time of an elliptical galaxy is far shorter than its age. Exceptionally, the phases whose temperatures become below $T_{\text {crit }}\left(=10^{5} \mathrm{~K}\right)$ are not considered to be in the pressure equilibrium because the condition of the pressure equilibrium will broken down for the phases due to the high cooling rate. They are assumed to cool immediately and drop out of the hot ISM. The gas left after galactic wind in the $j$ th zone is also considered as a phase called "zero-phase" or $(0, j, 0)$-th phase.

In $\$ 2.1$, we describe the formation of the phases in the $j$ th zone at $t=t_{i, j}(1 \leq i \leq n, 1 \leq j \leq m)$ unless otherwise mentioned. We will often omit the subscripts $i$ and $j$ in $\S 2.1$. In $\$ 2.2$, we describe the evolution of the phases and the galaxy.

\subsection{FORMATION OF EACH PHASE}

\subsubsection{Mass-Loss Phases}

We assume that the temperature of the gas ejected by the stellar wind immediately becomes equal to the stellar temperature of the galaxy $T \star(R)=\left[\sigma_{\star}(R)\right]^{2} \mu m_{H} / k_{B}$, where $\mu$ is the mean molecular weight $(=0.6), m_{\mathrm{H}}$ is the mass of the hydrogen atom, and $k_{\mathrm{B}}$ is Boltzmann constant.

Under this assumption, the initial temperature and density of the mass-loss phase are then given by

$$
\begin{gathered}
T^{(i, j, M L)}\left(t_{i, j}\right)=T_{\star}\left(R_{j}\right), \\
\rho^{(i, j, M L)}\left(t_{i, j}\right)=\frac{\mu m_{\mathrm{H}} P^{(j)}\left(t_{i, j}\right)}{k_{\mathrm{B}} T_{\star}\left(R_{j}\right)},
\end{gathered}
$$

where $P^{(j)}(t)$ is the average pressure of the ISM in the $j$ th zone. The pressure $P^{(j)}(t)$ is obtained by solving the evolution equations of the galaxy described in $\$ 2.2$.

The gas and iron mass of the mass-loss phase at its birth time, $M^{(i, j, M L)}\left(t_{i, j}\right)$ and $M_{\mathrm{Fe}}^{(i, j, M L)}\left(t_{i, j}\right)$, respectively, are given by

$$
M^{(i, j, M L)}\left(t_{i, j}\right)=\int_{t_{i-1, j}}^{t_{i, j}} L_{\star}^{(j, M L)}(t) d t
$$




$$
M_{\mathrm{Fe}}^{(i, j, M L)}\left(t_{i, j}\right)=\int_{t_{i-1, j}}^{t_{i, j}} L_{\mathrm{Fe}}^{(j, M L)}(t) d t
$$

where $L_{\star}^{(j, M L)}$ and $L_{\mathrm{Fe}}^{(j, M L)}$ are the gas and iron mass ejection rates from stars in the $j$ th zone, respectively. Since the time-scale of star formation in an elliptical galaxy, which is typically $10^{7-8}$ yr, is short enough compared with the galaxy age (e.g. Arimoto \& Yoshii 1987), we assume that the stellar system of the galaxy formed at $t=0$ simultaneously. Thus, $L_{\star}^{(j, M L)}$ and $L_{\mathrm{Fe}}^{(j, M L)}$ are given by

$$
\begin{gathered}
L_{\star}^{(j, M L)}(t)=f\left|\frac{d m(\tau)}{d \tau}\right|_{\tau=t} \phi(m(\tau=t)) M_{\star}^{(j)}(t), \\
L_{\mathrm{Fe}}^{(j, M L)}(t)=Z_{\mathrm{ML}} f\left|\frac{d m(\tau)}{d \tau}\right|_{\tau=t} \phi(m(\tau=t)) M_{\star}^{(j)}(t),
\end{gathered}
$$

where $\phi(m)$ and $Z_{\mathrm{ML}}$ are the initial mass function (IMF) and the iron abundance of the mass-loss gas, respectively. The power of the IMF is taken to be 1.35. Total mass of the stars in the $j$ th zone, $M_{\star}^{(j)}(t)$, is given by

$$
M_{\star}^{(j)}(t)=\int_{R_{j-1}(t)}^{R_{j}(t)} 4 \pi R^{2} \rho_{\star}(R) d R .
$$

The relation between the mass of a star $m(\tau)$, and its lifetime $\tau$, is given by

$$
\log _{10} m(\tau)=1.983-1.054 \sqrt{\log _{10} \tau+2.52}
$$

where the mass is in units of solar mass $\left(M_{\odot}\right)$ and the lifetime is in units of Gyr. (Larson 1974). Because we consider the evolution of the hot ISM after galactic wind stops $\left(t>t_{0}=0.5 \mathrm{Gyr}\right.$ ), this equation implies that we do not have to consider stars with mass larger than $2.85 M_{\odot}$ in our calculation period. We assume that stars with mass in the range of $0.1-2.85 M_{\odot}$ lose their masses by stellar winds ; we simply assume that the mass loss occurs instantaneously at the end of the life given by the fraction $f$ of the initial mass of a star with $m$,

$$
f= \begin{cases}0 & \text { for } m \leq 0.7 \\ 0.42 m & \text { for } 0.7<m \leq 1.0 \\ 0.8-0.43 / m & \text { for } 1.0<m \leq 2.85\end{cases}
$$

(Köppen \& Arimoto 1991).

Although the metal abundance distribution of the stars or mass-loss gas is not well-known beyond the effective radius, it is assumed to be

$$
Z_{\mathrm{ML}}(R)=Z_{\mathrm{ML}}\left(R_{e}\right)\left(R / R_{e}\right)^{-1 / 2}
$$

(Arimoto et al. 1997), where $R_{e}$ is the effective radius and we assume that $R_{e}=12 R_{a \star}$.

The density $\rho^{(i, j, M L)}\left(t_{i, j}\right)$, the temperature $T^{(i, j, M L)}\left(t_{i, j}\right)$, the mass $M^{(i, j, M L)}\left(t_{i, j}\right)$, and the iron mass $M_{\mathrm{Fe}}^{(i, j, M L)}\left(t_{i, j}\right)$ determined by above equations give the initial conditions of the evolution equations of the phases (see $\$ 2.2$ ). 


\subsubsection{Bored Shell and Mixed Cavity Phases}

Next, we describe the formation of the shell and cavity phases. We consider only Type Ia supernovae (SN Ia) because we are concerned with the evolution of the hot ISM after galactic wind stops.

In Paper I, we assumed that the SNRs consist of two parts, outer shell and inner cavity region, and that they evolve as separate phases. We called the former "shell phase" and the latter "cavity phase", denoted by the indices $s$ and $c$, respectively. When the distance from the center of the SNR is $r$ and the SNR radius is $r_{\mathrm{s}}$, which is referred as "shock front radius" in Paper I, the inner cavity region and the outer shell region correspond to $r<(1-k) r_{\mathrm{s}}$ and $(1-k) r_{\mathrm{s}}<r<r_{\mathrm{s}}$, respectively, where $k$ is representing the width of the shell. The SNR radius is given by

$$
\begin{aligned}
r_{\mathrm{S}} & =\left[\frac{8}{25(\gamma+1)}\right]^{1 / 3}\left(P^{(j)}\right)^{-1 / 3}\left(2.02 E_{\mathrm{SN}}\right)^{1 / 3} \\
& \sim 70\left(\frac{P^{(j)}}{10^{5} \mathrm{~cm}^{-3} \mathrm{~K}}\right)^{-1 / 3}\left(\frac{E_{\mathrm{SN}}}{10^{51} \mathrm{erg}}\right)^{1 / 3} \mathrm{pc},
\end{aligned}
$$

where $\gamma(=5 / 3)$ is adiabatic constant, and $E_{S N}\left(=10^{51} \mathrm{erg}\right)$ is the energy released from a supernova (Paper I).

The density, temperature, and mass of the shell and cavity region before the cavity floats by buoyant force are

$$
\begin{aligned}
\rho_{s}=\int_{(1-k) r_{\mathrm{s}}}^{r_{\mathrm{s}}} 4 \pi \rho_{\mathrm{Sd}}(r) r^{2} d r / \int_{(1-k) r_{\mathrm{s}}}^{r_{\mathrm{s}}} 4 \pi r^{2} d r \\
\rho_{\mathrm{c}}=\frac{3 m_{\mathrm{c}}}{4 \pi(1-k)^{3} r_{\mathrm{s}}^{3}}, \\
T_{\mathrm{s}}=\frac{2}{3} \frac{\mu m_{\mathrm{H}} U_{\mathrm{s}}}{k_{\mathrm{B}}}+\hat{T}^{(j)} \\
T_{\mathrm{c}}=\frac{2}{3} \frac{\mu m_{\mathrm{H}} U_{\mathrm{c}}}{k_{\mathrm{B}}}+\hat{T}^{(j)} \\
m_{\mathrm{s}}=\rho_{\mathrm{s}} \int_{(1-k) r_{\mathrm{s}}}^{r_{\mathrm{s}}} 4 \pi r^{2} d r \\
m_{\mathrm{c}}=m_{\mathrm{SNR}}-m_{\mathrm{s}}
\end{aligned}
$$

respectively, where $\rho_{\mathrm{Sd}}(r)$ is the density distribution of the Sedov solution, $U$ is the specific thermal energy, $\hat{T}^{(j)}$ is the average temperature of the ISM in the $j$ th zone, and $m_{\mathrm{SNR}}$ is the mass inside the SNR radius. Their derivations are described in Paper I. Note that there is no physical meaning in using Sedov solution ; it is only an approximation of Fig.2 - 4 of Mathews (1990) as we discussed in Paper I. 
In Paper I, we ignored floating of the hot cavity region by buoyant force and subsequent mixing by Rayleigh-Taylor instability (buoyant mixing) for simplicity, although Mathews (1990) indicated that it is effective. In this paper, we consider the effect as the followings.

The time-scale for the cavity region to move by one diameter and to mix into the local ISM is $\lesssim 10^{6-7}$ yr (Mathews 1990). Since it is less than the time-scale of the evolution of elliptical galaxies, we assume that the cavity region moves by one diameter and mixes into the local ISM immediately after its birth. Figure 1 gives an outline. The region, $A+B+C$, that is, the local ISM, part of the shell region, and the cavity region, is assumed to mix uniformly. Hereafter, we refer to the region $A+B+C$ as the mixed cavity region, and refer to the region $D$ as the bored shell region, denoted by the indices $m c$ and $b s$, respectively. They evolve as separate phases. We call the former "mixed cavity phases (MCPs)" and the latter "bored shell phases (BSPs)".

The density, temperature, and mass of the bored shell region and mixed cavity region are

$$
\begin{gathered}
\rho_{b s}=\rho_{s}, \\
\rho_{m c}=\frac{m_{c}+\rho_{s} V_{\mathrm{B}}+\rho^{(j)} V_{\mathrm{A}}}{V_{\mathrm{A}}+V_{\mathrm{B}}+V_{\mathrm{C}}} \\
T_{b s}=T_{s} \\
T_{\mathrm{mc}}=\frac{2}{3} \frac{\mu m_{\mathrm{H}}}{k_{\mathrm{B}}} \frac{E_{\mathrm{SN}}-m_{b s} U_{s}}{m_{m c}}+\hat{T}^{(j)}, \\
m_{b s}=\rho_{s} V_{\mathrm{B}} \\
m_{m c}=m_{c}+\rho_{s} V_{\mathrm{B}}+\rho^{(j)} V_{\mathrm{A}},
\end{gathered}
$$

where $V_{\mathrm{A}}, V_{\mathrm{B}}$, and $V_{\mathrm{C}}$ are the volumes of the region $A, B$, and $C$, respectively, and $\rho^{(j)}$ is the average density of the $j$ th zone.

In Paper I, we also assumed that the metal ejected from a Type Ia supernova is uniformly dispersed within the SNR radius. However, Jun, Jones, \& Norman (1996) showed that most of the metal is trapped in the cavity region even in inhomogeneous medium. Thus, in this paper, we assume that the metal ejected from a Type Ia supernova is trapped in the mixed cavity region.

After the buoyant mixing, the iron mass in the bored shell and mixed cavity region are

$$
\begin{gathered}
m_{\mathrm{Fe}, b s}=m_{b s} \hat{Z}^{(j)}, \\
m_{\mathrm{Fe}, m c}=m_{\mathrm{Fe}}+m_{m c} \hat{Z}^{(j)},
\end{gathered}
$$

where $m_{\mathrm{Fe}}$ is the iron mass ejected by a supernova and $\hat{Z}^{(j)}$ is the average iron abundance of the ISM occupied by the SNR.

The densities and temperatures determined so far are adopted as the initial values of the BSP and MCP for the evolution equations of the phases described in $\$ 2.2$ as follows :

$$
\rho^{(i, j, b s)}\left(t_{i, j}\right)=\rho_{b s},
$$




$$
\begin{aligned}
T^{(i, j, b s)}\left(t_{i, j}\right) & =T_{b s}, \\
\rho^{(i, j, m c)}\left(t_{i, j}\right) & =\rho_{m c}, \\
T^{(i, j, m c)}\left(t_{i, j}\right) & =T_{m c} .
\end{aligned}
$$

They are determined uniquely if $E_{\mathrm{SN}}, m_{\mathrm{pro}}, m_{\mathrm{Fe}}, k, \rho^{(j)}, \hat{T}^{(j)}\left(=\mu m_{\mathrm{H}} P^{(j)} / \rho^{(j)}\right)$, and $\hat{Z}^{(j)}$ are given, where $m_{\text {pro }}$ is the mass of the progenitor star and is included by $m_{\mathrm{SNR}}$ We fix $E_{\mathrm{SN}}$, $m_{\text {pro }}$, and $m_{\mathrm{Fe}}$ by giving typical values, that is, $m_{\mathrm{pro}}=1.4 M_{\odot}, m_{\mathrm{Fe}}=0.5 \mathrm{M}_{\odot}$, and $E_{\mathrm{SN}}=10^{51}$ erg. We also fix $k=0.3$. The other parameters, $\rho^{(j)}, \hat{T}^{(j)}$, and $\hat{Z}^{(j)}$ are time-dependent and determined by solving the evolution equations of the galaxy described in $\$ 2.2$. For examples, for $\rho^{(j)}=1.67 \times 10^{-26} \mathrm{~g} \mathrm{~cm}^{-3}, \hat{T}^{(j)}=10^{7} \mathrm{~K}$, and $\hat{Z}^{(j)}=1.0 \mathrm{Z} \odot$, we can estimate each quantity : $\rho_{b s}=2.4 \times 10^{-26} \mathrm{~g} \mathrm{~cm}^{-3}, \rho_{m c}=8.2 \times 10^{-27} \mathrm{~g} \mathrm{~cm}^{-3}, T_{b s}=1.5 \times 10^{7} \mathrm{~K}, T_{m c}=1.5 \times 10^{7} \mathrm{~K}$, $m_{\mathrm{Fe}, \mathrm{bs}} / m_{b s}=1 \mathrm{Z} \odot$, and $m_{\mathrm{Fe}, \mathrm{mc}} / m_{m c}=2.9 \mathrm{Z} \odot$. As one can easily calculate from these values, the pressures of the MCP, BSP, and circumference ISM are not identical, that is, they have not been in pressure equilibrium yet. Therefore, we once regard equations (29) - (32) as the values at $t=t_{i-1, j}$ and as the initial conditions of the iteration to derive the values at $t=t_{i, j}$ (see the remark after equation (56)). Thus, after the iteration, the MCP, BSP, and circumference ISM are in pressure equilibrium. Although the cooling time of the MCP is shorter than that of the cavity phase in Paper I, it is longer than that of the shell phase in Paper I. This means that more iron ejected by supernova explosion remains in hot ISM until $t=t_{f}$ than in Paper I. The emission from the MCP have influence on X-ray spectrum (see $\$(3)$ ).

The gas and iron mass of the BSP and MCP at their birth time are given by

$$
\begin{aligned}
M^{(i, j, b s)}\left(t_{i, j}\right) & =\int_{t_{i-1, j}}^{t_{i, j}} L_{\star}^{(j, b s)}(t) d t, \\
M^{(i, j, m c)}\left(t_{i, j}\right) & =\int_{t_{i-1, j}}^{t_{i, j}} L_{\star}^{(j, m c)}(t) d t, \\
M_{\mathrm{Fe}}^{(i, j, b s)}\left(t_{i, j}\right) & =\int_{t_{i-1, j}}^{t_{i, j}} L_{\mathrm{Fe}}^{(j, b s)}(t) d t, \\
M_{\mathrm{Fe}}^{(i, j, m c)}\left(t_{i, j}\right) & =\int_{t_{i-1, j}}^{t_{i, j}} L_{\mathrm{Fe}}^{(j, m c)}(t) d t,
\end{aligned}
$$

where $L_{\star}^{(j, b s)}, L_{\star}^{(j, m c)}, L_{\mathrm{Fe}}^{(j, b s)}$, and $L_{\mathrm{Fe}}^{(j, m c)}$ are the mass and iron production rates of the BSP and MCP in the $j$ th zone, respectively ; they are given by

$$
\begin{gathered}
L_{\star}^{(j, b s)}(t)=r_{\mathrm{SN}}(t) m_{b s}(t) M_{\star}^{(j)}(t), \\
L_{\star}^{(j, m c)}(t)=r_{\mathrm{SN}}(t) m_{m c}(t) M_{\star}^{(j)}(t), \\
L_{\mathrm{Fe}}^{(j, b s)}(t)=r_{\mathrm{SN}}(t) m_{\mathrm{Fe}, b s}(t) M_{\star}^{(j)}(t), \\
L_{\mathrm{Fe}}^{(j, m c)}(t)=r_{\mathrm{SN}}(t) m_{\mathrm{Fe}, m c}(t) M_{\star}^{(j)}(t),
\end{gathered}
$$

where $r_{\mathrm{SN}}(t)$ is the SN Ia rate per unit stellar mass. The time dependence of the SN Ia rate is assumed to be $r_{\mathrm{SN}}(t) \propto t^{-0.5}$ (David, Forman, \& Jones 1990). The normalization is given by the SN Ia rate at $t=t_{f}$, which is specified later. 


\subsubsection{Can Magnetic Field Suppress Mixing of the Phases?}

Nulsen (1986) pointed out that perturbations can be pinned in cooling flows when magnetic stresses are able to suppress their relative motions. This occurs when the Alfven speed, $v_{\mathrm{A}}$ is larger than the terminal velocity, $v_{t}$ defined by the force balance between ram pressure and the excess gravity. Hattori et al. (1995) confirm this numerically; they show that magnetic field can suppress relative motion in a non-linear perturbation and can help the growth of thermal instability as long as the size of perturbation is smaller than the critical value

$$
\lambda_{\text {crit }}=1.5\left(\frac{\delta}{10}\right)^{-1}\left(\frac{\beta}{350}\right)^{-1}\left(\frac{L}{5 \mathrm{kpc}}\right) \mathrm{pc},
$$

where $\beta$ is the ratio of the gas pressure to the magnetic pressure, and $L$ is the scale height of pressure. The density contrast, $\delta$, is defined as

$$
\delta=\frac{\rho-\rho_{b}}{\rho_{b}}
$$

where $\rho$ and $\rho_{b}$ are the density of the perturbation and unperturbed flow, respectively.

For the case of a temperature of $10^{7} \mathrm{~K}$ and a density of $\rho_{b}=1.67 \times 10^{-26} \mathrm{~g} \mathrm{~cm}^{-3}$, and there is a $1 \mu G$ magnetic field, one finds that $\beta=350$ and $r_{s}=70 \mathrm{pc}$; in the following argument in this subsection, we will use these parameters and take $L=5 \mathrm{kpc}$. When $k=0.3$, spatial scale lengths of cavity and shell region respectively are $50 \mathrm{pc}$ and $20 / 2=10 \mathrm{pc}$, following the definition of Hattori et al. (1995). Since the initial value of $\delta$ for the shell region is less than one, the critical wavelength is $\lambda_{\text {crit }}>15 \mathrm{pc}$. This indicates that the shell region can be supported by magnetic field before the cooling becomes efficient. On the contrary, one can show that the cavity region, before mixing, cannot be supported, if equation (41) can be applied to less dense perturbation for $|\delta| \sim 1$; it will move upwards and mix with the ambient medium as we assumed in $\$ 2.1 .2$.

As each phase cools, the density contrast increases. Hattori \& Habe (1990) show that if magnetic tension supports a perturbation until $\delta>10$, the gas in the perturbation can cool to $10^{4} \mathrm{~K}$. From equation (41), one finds $\lambda_{\text {crit }}=1.5 \mathrm{pc}$ when $\delta=10$. Since the spatial scale length of gas blobs composing a mass-loss phase is typically $\sim 1$ pc (Mathews 1990), the blobs can cool to $10^{4} \mathrm{~K}$ without mixing and drop out of hot ISM. On the other hand, since the spatial scale lengths of mixed cavity region and bored shell region are 50 and $10 \mathrm{pc}$, respectively, they cannot cool to $10^{4} \mathrm{~K}$ without mixing unless they fragment into small pieces before the cooling becomes effective ( $~\left(10^{7-8} \mathrm{yr}\right.$ ). However, Jun et al. (1996) show that a SNR in inhomogeneous medium has complicated structure ; thus, we do not think that each region evolves as one gas blob for a long time. Moreover, the estimation of Hattori \& Habe (1990) does not include the effect of metal abundance fluctuation which makes gas blobs with higher metal abundance cool faster, and makes the value of $\delta$ until which magnetic tension should support them smaller. Furthermore, since a SNR amplifies magnetic field (Jun \& Norman 1996), $\lambda_{\text {crit }}$ may be large around it. Thus, 
we assume in $\S 3$ that the MCP and BSP also comove with ambient ISM, although we consider an additional model in which the MCP and BSP mix with ambient ISM in wider region.

The above estimations may be too simple ; strength variation and structure of magnetic fields may affect the evolutions of gas blobs. However, since a full treatment of convection in inhomogeneous medium would be very difficult, we consider comoving flows as a first-step of the research.

\subsection{EVOLUTION OF THE PHASES AND THE GALAXY}

The energy equation for the $(i, j, \alpha)$-th phase for $t>t_{i, j}$ is given by

$$
\frac{\rho^{(i, j, \alpha)}(t)}{\gamma-1} \frac{d}{d t}\left(\frac{k_{\mathrm{B}} T^{(i, j, \alpha)}(t)}{\mu m_{\mathrm{H}}}\right)-\frac{k_{\mathrm{B}} T^{(i, j, \alpha)}(t)}{\mu m_{\mathrm{H}}} \frac{d}{d t} \rho^{(i, j, \alpha)}(t)=-\left(n_{e}^{(i, j, \alpha)}\right)^{2} \Lambda\left(Z^{(i, j, \alpha)}, T^{(i, j, \alpha)}(t)\right)
$$

where $n_{e}^{(i, j, \alpha)}$ is the electron density of the $(i, j, \alpha)$-th phase and $\Lambda$ is the cooling function approximated by

$$
\begin{aligned}
& \Lambda\left(Z^{(i, j, \alpha)}, T^{(i, j, \alpha)}\right)=\left[2.1 \times 10^{-27}\left(1+0.1 \frac{Z^{(i, j, \alpha)}}{\mathrm{Z}_{\odot}}\right)\left(\frac{T^{(i, j, \alpha)}}{\mathrm{K}}\right)^{0.5}\right. \\
& \left.+8.0 \times 10^{-17}\left(0.04+\frac{Z^{(i, j, \alpha)}}{\mathrm{Z}_{\odot}}\right)\left(\frac{T^{(i, j, \alpha)}}{\mathrm{K}}\right)^{-1.0}\right] \\
& \left(\operatorname{ergs~cm}{ }^{-3} \mathrm{~s}^{-1}\right) \text {. }
\end{aligned}
$$

This is an empirical formula derived by fitting to the cooling curves calculated by Böhringer \& Hensler (1989).

In our model, we assume that all existing phases in a certain zone have same pressure. Thus, for $t_{i, j}<t_{l, j}$,

$$
\rho^{(i, j, \alpha)}\left(t_{l, j}\right) \frac{k_{\mathrm{B}} T^{(i, j, \alpha)}\left(t_{l, j}\right)}{\mu m_{\mathrm{H}}}=P^{(j)}\left(t_{l, j}\right) .
$$

We assume that the ISM is in hydrostatic equilibrium, that is,

$$
\begin{gathered}
\frac{\Delta P^{(j)}}{\Delta R_{j}}=-\rho^{(j)} \frac{G M\left(R_{j}\right)}{\left(R_{j}\right)^{2}}, \\
\rho^{(j)}\left(t_{l, j}\right)=\frac{M_{\mathrm{g}}^{(j)}\left(t_{l, j}\right)}{V^{(j)}\left(t_{l, j}\right)},
\end{gathered}
$$

where $M_{\mathrm{g}}^{(j)}$ and $V^{(j)}$ are respectively the total gas mass and volume of the $j$ th zone, $\Delta P^{(j)}=P^{(j+1)}-P^{(j)}$, and $\Delta R_{j}=R_{j}-R_{j-1}$. We define $P^{(j)}$ as the pressure just outside $R_{j-1}$. 
The gravitational mass within $R$ is given by

$$
M(R)=\int_{0}^{R} 4 \pi R^{2}\left(\rho_{\star}+\rho_{h}\right) d R .
$$

The total gas mass, the iron mass, and the gas volume of the $j$ the zone are the summation of those of each phase ;

$$
\begin{aligned}
M_{\mathrm{g}}^{(j)}(t) & =\sum_{\alpha} \sum_{i, T^{(i, j, \alpha)}>T_{\mathrm{crit}}} M^{(i, j, \alpha)}(t), \\
M_{\mathrm{g}, \mathrm{Fe}}^{(j)}(t) & =\sum_{\alpha} \sum_{i, T^{(i, j, \alpha)}>T_{\mathrm{crit}}} M_{\mathrm{Fe}}^{(i, j, \alpha)}(t), \\
V^{(j)}(t) & =\sum_{\alpha} \sum_{i, T^{(i, j, \alpha)}>T_{\mathrm{crit}}} V^{(i, j, \alpha)}(t),
\end{aligned}
$$

where

$$
V^{(i, j, \alpha)}(t)=M^{(i, j, \alpha)}(t) / \rho^{(i, j, \alpha)}(t) .
$$

As mentioned above, the phases whose temperatures are below $T_{\text {crit }}\left(=10^{5} \mathrm{~K}\right)$ are not included in the summation.

Note that part of the SNRs is composed of pre-existing phases ; the masses of the pre-existing phases are reduced by the occupation by the SNRs. Thus, the mass and iron mass of the pre-existing $(i, j, \alpha)$-th phase at $t=t_{l, j}\left(t_{i, j}<t_{l, j}\right)$ are given by

$$
\begin{gathered}
M^{(i, j, \alpha)}\left(t_{l, j}\right)=M^{(i, j, \alpha)}\left(t_{l-1, j}\right)-\left.M_{\mathrm{SNR}, l}^{(j)} \frac{V^{(i, j, \alpha)}(t)}{V^{(j)}(t)}\right|_{t=t_{l-1, j}}, \\
M_{\mathrm{Fe}}^{(i, j, \alpha)}\left(t_{l, j}\right)=M_{\mathrm{Fe}}^{(i, j, \alpha)}\left(t_{l-1, j}\right)-\left.M_{\mathrm{SNR}, l}^{(j)} \frac{M_{\mathrm{Fe}}^{(i, j, \alpha)}(t)}{M^{(i, j, \alpha)}(t)} \frac{V^{(i, j, \alpha)}(t)}{V^{(j)}(t)}\right|_{t=t_{l-1, j}},
\end{gathered}
$$

respectively, where $M_{\mathrm{SNR}, l}^{(j)}$ is the mass occupied by SNRs during $t_{l-1, j}<t<t_{l, j}$, and is given by

$$
M_{\mathrm{SNR}, l}^{(j)}=\int_{t_{l-1, j}}^{t_{l, j}} \rho^{(j)}(t) V_{S} r_{\mathrm{SN}}(t) M_{\star}^{(j)}(t) d t
$$

where $V_{\mathrm{S}}=V_{\mathrm{A}}+V_{\mathrm{B}}+V_{\mathrm{C}}+V_{\mathrm{D}}$.

We take the sound crossing time as the time-steps for the calculations,

$$
t_{l+1, j}-t_{l, j}=\Delta R_{j}\left(t_{l, j}\right)\left(\gamma \frac{k_{\mathrm{B}} \hat{T}^{(j)}}{\mu m_{\mathrm{H}}}\right)^{-1 / 2}
$$

We derive $\rho^{(i, j, \alpha)}\left(t_{l, j}\right)$ and $T^{(i, j, \alpha)}\left(t_{l, j}\right)$ from $\rho^{(i, j, \alpha)}\left(t_{l-1, j}\right)$ and $T^{(i, j, \alpha)}\left(t_{l-1, j}\right)$ by iterating Eqs.(43), (45), (46), (49), (51), and (53) until they converge. 


\section{MODEL RESULTS}

In this section, we present results of the evolution equations described above. We reduce the number of the free parameters by giving typical fixed values to some of them. First, for the parameters regarding the whole galaxy, we take $t_{0}=0.5 \mathrm{Gyr}, t_{f}=10 \mathrm{Gyr}$.

We assume that the gas distribution and abundance distribution at $t=t_{0}$ are

$$
\begin{gathered}
\rho_{g a s}(R)=\rho_{0 g a s}\left[1+\left(R / R_{a \star}\right)^{2}\right]^{-3 / 2}, \\
Z_{0}(R)=Z_{0}\left(R_{e}\right)\left(R / R_{e}\right)^{-1 / 2}
\end{gathered}
$$

for $R<R_{t}$, respectively. The normalization, $\rho_{0 \text { gas }}$, is determined by assuming that the total gas mass at $t=t_{0}$ is $1 / 30$ of the total stellar mass. The normalization, $Z_{0}\left(R_{e}\right)$, is a parameter. We give the initial zone radius so that enough spatial resolution is obtained at $t=t_{f}$. The initial number of the zone, $m$, is 30 . We can write

$$
\begin{gathered}
V^{(0, j, 0)}\left(t_{0}\right)=V^{(j)}\left(t_{0}\right)=\frac{4 \pi}{3}\left[R_{j}\left(t_{0}\right)^{3}-R_{j-1}\left(t_{0}\right)^{3}\right] \\
M^{(0, j, 0)}\left(t_{0}\right)=M_{\mathrm{g}}^{(j)}\left(t_{0}\right)=\int_{R_{j-1}\left(t_{0}\right)}^{R_{j}\left(t_{0}\right)} 4 \pi R^{2} \rho_{g a s}(R) d R \\
M_{\mathrm{Fe}}^{(0, j, 0)}\left(t_{0}\right)=M_{\mathrm{Fe}, \mathrm{g}}^{(j)}\left(t_{0}\right) .
\end{gathered}
$$

We take the temperatures of the zero-phases

$$
T^{(0, j, 0)}\left(t_{0}\right)=T_{\star}\left(R_{j}\left(t_{0}\right)\right) .
$$

We solve the basic equations described in $\$ 2$ for the galaxy models whose details are given in Table 1 . The SN Ia rate is normalized by the values at $t=t_{f}(=10 \mathrm{Gyr})$ shown in Table 1. The values are expressed in units of $\mathrm{SNu}$, that is, the number of $\mathrm{SNe}$ per $10^{10} h^{-2} \mathrm{~L}_{\mathrm{B} \odot}$ per $100 \mathrm{yr}$ $\left(H_{0}=100 h \mathrm{~km} \mathrm{~s}^{-1} \mathrm{Mpc}^{-1}\right.$; we set $\left.h=0.5\right)$. The ratio of the mass to luminosity is taken to be $8 M_{\odot} / L_{\odot}$, and the rate for SN Ia is normalized by using this ratio. Models A3-A6 and B3-B6 are calculated to see the effect of metal-abundance distribution for the mass-loss gas. In these models, the mass-loss phase born in each time-step is divided into two phases with equal masses and different abundances corresponding to the two figures for $Z_{\mathrm{ML}}\left(R_{e}\right)$ (Table 1). On the other hand, in models A1 and A2, each component has the same abundance or the mass-loss gas has one kind of the abundance. In model A5, shell and cavity regions mix into circumference ISM of 26 times their volumes, respectively. This means that shell and cavity region mix into circumference ISM in wider region than other models.

Figure 2(a) shows the time evolution of $R_{j}(1 \leq j \leq m)$ in models A1 and A2 ; this figure shows the influence of SN Ia on the gas evolution. Cooling flow is established in the inner region. 
Note that in other models, the cooling flow is also established. Although the supernova rate of model A2 is larger than that of model A1, the inward velocity in model A2 is not reduced. This fact indicates that supernovae are not effective heating sources of the ISM in the inner region of the galaxy. The reason is that the $\mathrm{BCP}$ and MCP cool effectively owing to their high metal abundance (see $\S(2.1 .2)$, and that they have large thermal energies when they are born ; the sum of their energy is

$$
E_{b s}+E_{m c}=E_{\mathrm{SN}}+\frac{3 k \hat{T}}{2 \mu m_{\mathrm{H}}} \rho V_{\mathrm{S}}>E_{\mathrm{SN}}
$$

except for models A5 and B5. This indicates that more energy than that supplied by supernova explosion radiates from the BSP and MCP in a short time. Thus, supernovae cannot heat the ISM effectively. In outer region, the outflow velocity in model A2 is larger that in model A1; supernovae are effective as heating sources. In this region, cooling times of the BSP and MCP are so long that they do not radiate their thermal energy so much. Thus, the energy released by supernovae is transfered to the circumferential ISM before the cooling.

In Figure 2(b), outward velocity of model A5 is larger than that of model A3. This is because mixing of SNRs makes their cooling time longer. Therefore, the energy transition between SNRs and circumferential ISM is more effective in model A5.

Figures 3 - 5 show the distributions of the density, luminosity averaged temperature, and metal abundance at $t=t_{f}$ (at $t=9.4$ Gyr for model A5 because the radius of the innermost zone is $\sim 20 \mathrm{kpc}$ at $t=t_{f}$ ). We confirmed that, for models in Paper I, the luminosity averaged temperatures are almost same as those derived by spectral simulations which we did in Paper I. On the contrary, the luminosity averaged metal abundances are not consistent with those derived by the spectral simulations. Thus in this section, we simply use $Z_{m}=M_{\mathrm{g}, \mathrm{Fe}}^{(j)} / M_{\mathrm{g}}^{(j)}$ as the metal abundance, and later, derive X-ray spectrum of the model galaxies. The units of metal abundance is the solar abundance $\mathrm{Z}_{\odot}, 1.7 \times 10^{-3}$. Since our model does not have enough spatial resolution in the central region of the galaxy, we infer the distributions of the density, luminosity averaged temperature, and metal abundance by presenting the values of the zones which disappear just before $t=t_{f}$. The values shown in Table 2 are the ones when the zone radii are $\sim 0.3 \mathrm{kpc}$. For model A2, we stopped calculating the evolution of the innermost zone and deleted it before its radius decreases to $0.3 \mathrm{kpc}$. This is because masses of some phases in the innermost zone become negative by equation (53), because of large $M_{\mathrm{SNR}, l}^{(j)}$ and density contrast among phases. In order to overcome this defect of our model, the exact treatment of the evolution of supernova remnants in inhomogeneous medium is required. However, we think this deletion does not affect the evolution of the outer zones, because when we stop the calculation, the volume of the innermost zone is far smaller than those of the outer zones.

Figure 3 show that the density varies crudely as $\rho \propto R^{-1.5}$ for $R \gtrsim 1 \mathrm{kpc}$. The distributions of density are almost independent of the models. This reflects that the density distributions are mainly decided by energy injection rate and vary as $\propto \rho_{\star}^{1 / 2}$ (Sarazin, \& White 1987). The high central density of models A5 and B5 reflects small density contrast among the phases in the 
innermost zones (Table 2).

The temperature gradually rises toward the galactic center (Figure 4). This temperature rise is caused by adiabatic heating owing to the gas inflow (Figure 2) ; in our models, the phases with low-cooling rates are brought to the center and compressed, because high-cooling phases drop out and the volume of the inner zones reduces. In the central region, the temperature falls down (Table 2). In this region, all phases start to cool because of high pressure.

The metal abundances, $Z_{m}$, are very different among the models A1-A3 (Figure 5(a)). We show the metal abundance distribution of mass-loss gas when $Z_{\mathrm{ML}}\left(R_{e}\right)=1 Z_{\odot}$ (equation (12)) in Figure 5 for reference. In model A2, the high supernova rate makes the metal abundance high. This high metal abundance is inconsistent with the observations (Awaki et al. 1994 ; Loewenstein et al. 1994 ; Matsushita et al. 1994 ; Mushotzky et al. 1994 ; Kim \& Fabbiano 1995 ; Davis \& White 1996 ; Matsumoto et al. 1996). This may indicate that the real supernova rate is lower than that derived by the observation $(\sim 0.2 \mathrm{SNu}$; Tammann 1982$)$. In the central region, high-abundance phases generally cool and drop out of hot ISM faster than other phases, although it depends on their temperatures and densities. This makes the average metal abundance in that region small. However, since the negative metal abundance gradient of the stars or mass-loss gas cancels this effect, the metal abundance of the hot ISM is constant or rises toward the center of the model galaxy except for model A6 and B6 (Figure 5). Since this result depends on the assumption of equation (12), the observation of the metal abundance of the stars beyond the effective radius is encouraged. Moreover, gas flows inward from the outer region where the metal abundance of mass-loss gas is small. This means that as gas ejection rate of the stellar system decreases, mass fraction of low-abundance phases born in the outer region increases in a galaxy. In the outer region, the selective cooling is not effective and the metal abundances of the hot ISM directly reflects the metal injection from stars including supernovae.

The metal abundance in models A3 is smaller than those in models A1 (Figure 5(a)), because the mass-loss phases with higher metal abundance cool faster than those with lower metal abundance. Compared with model A3, model A4 shows higher abundance (Figure 5(a) and 5(b)). In model A4, part of high abundance gas of the zero-phase is occupied by SNRs which survive until $t=t_{f}$. In model A6 and B6, mass-loss phases with low metal abundance dominate in the central region at $t=t_{f}$.

Although the metal abundances of the models except for A2 is low, they cannot be compared directly with the $A S C A$ results $\left(\lesssim 0.5 \mathrm{Z}_{\odot}\right)$. Since the density of phases with high abundance are generally high, emission from them can affect X-ray spectrum. Therefore, we simulate the spectra at $t=t_{f}$, as we did in Paper I, for models A3 - A6. We simulate the spectra of innermost zones and those of zones at $\sim 20 \mathrm{kpc}$. Although we do not consider projection effect, contamination of outer zones is less than $20 \%$ for the innermost zones. For $\gtrsim 20 \mathrm{kpc}$, the variation among zones are little except for densities. The distance to the model galaxies is assumed to be $15 \mathrm{Mpc}$. We use XSPEC package (version 9.00) and response function of $A S C A$ Solid-State Imaging Spectrometer 
(SIS). The model spectrum are fitted by Meka model (Mewe, Gronenschild, and van den Oord 1985 ; Mewe, Lemen, and van den Oord 1986 ; Kaastra 1992). The method is described in Paper I.

The results are shown in Figure 6. Because of the low luminosity, $Z_{s p}$ at $\sim 20 \mathrm{kpc}$ has an error of $\sim 0.4 Z_{\odot}$. We present the spectrum of the innermost zone of model $\mathrm{A} 4$ as an example (Figure 7). The central metal abundance derived by spectral fitting, $Z_{s p}$ is larger than $Z_{m}$ for models $\mathrm{A} 3$ and A4. This is because that MCPs affect the X-ray spectrum by their strong metal emission. The number of MCP which can survive until $t=t_{f}$ is small compared with those of other phases; for example, for the innermost zone in model A4 the ratio is $M L L: M L H: B S P: M C P=161: 14: 146: 97$, where $M L L$ and $M L H$ refer to mass-loss phase with low and high metal abundance, respectively. On the contrary, the luminosity ratio is $M L L: M L H: B S P: M C P \sim 2: 1: 4: 4$. Note that for the zone at $\sim 20 \mathrm{kpc}$, the luminosity ratio is $M L L: M L H: B S P: M C P \sim 2: 3: 2: 2$. In model A5, $Z_{s p} \sim Z_{m}$ in the galactic center, because of small abundance fluctuation among the phases. However, the luminosity of MCP and BSP dominates that of mass-loss phase as models A3 and A4.

In the central region, the derived metal abundances, $Z_{s p}$, are larger than the $A S C A$ results (Mushotzky et al. 1994) except for model A6, although $Z_{s p} \lesssim Z_{\mathrm{ML}}$ (Figure 6). This may indicate that our model is too simple to predict metal abundance quantitatively. Alternatively, supernova explosion rate in elliptical galaxies may be $<0.01 \mathrm{SNu}$; the central abundance deficit of NGC 4472 (Irwin \& Sarazin 1996) may correspond to that in models A6 and B6. As discussed above, our model predicts that in a central region of a galaxy, iron line emission from SNRs of Type Ia supernovae, if any, should be prominent. That is, observed [Si/Fe] and $[\mathrm{Mg} / \mathrm{Fe}]$ should decrease towards the center.

The X-ray luminosities of the model galaxies are $\sim 10^{41-42} \mathrm{erg} \mathrm{s}^{-1}$ for $t \sim 10^{10} \mathrm{yr}$; the luminosities fluctuate because of the small number of the zone.

\section{SUMMARY AND CONCLUSIONS}

In this paper, we have presented a model of the evolution of the hot gas for elliptical galaxies after galactic wind period under the assumption that the gases ejected from stars do not mix with the circumferential ISM globally. The ejected gases evolve separately according to their birth time, position, and origin. We considered three origins of the ejected gas, that is, shell and cavity of supernova remnants and mass-loss gas. Furthermore, we considered the floating of the cavity and subsequent mixing by Rayleigh-Taylor instability.

The main results and conclusions can be summarized as follows:

1. The model predicts that the supernovae are not effective as heating sources of the ISM in the 
inner region of galaxies after the galactic wind stops. In the inner region, supernova remnant can cool rapidly because of their high density and/or metal abundance. Since the remnants initially have large thermal energy, the energy ejected by supernova explosions is radiated and supernovae do not heat up the ISM. Thus, cooling flow is established even if supernova rate is large. In the outer region of the galaxies, the cooling time of the remnants is long. Thus, most of energy ejected by supernova explosions is not radiated and it is transfered into the circumferential ISM. Mixing of SNRs with ambient ISM makes this transfer more effective.

2. In a inner region of a galaxy, the present iron abundance of the hot ISM can be less than that of the mass-loss gas or stars if the supernova rate is small, because the phases with higher metal abundance generally cool faster and gas inflows from outer region where the metal abundance of the mass-loss gas is small. However, the spectral simulations show that predicted metal abundances are still larger than the ones observed by $A S C A$ in the central region, if the present supernova rate is $\gtrsim 0.01 \mathrm{SNu}$. In the outer region where the selective cooling is ineffective, metal abundance of the ISM directly reflects that of the gas ejected from stars. Our model predicts that iron line emission by SNRs is prominent in the central region, and that $[\mathrm{Si} / \mathrm{Fe}]$ and $[\mathrm{Mg} / \mathrm{Fe}]$ decrease towards the galactic center.

We thank H. Kodama, N. Gouda, H. Sato, M. Hattori, Y, Ikebe, H. Susa, and T. Kan-ya for useful discussion and comments. We appreciate K. Koyama for using a computer. We are also

grateful to H. Matsumoto and E. Rokutanda for providing ASCA data. This work was supported in part by the JSPS Research Fellowship for Young Scientists.

\section{REFERENCES}

Arimoto, N., Matsushita, K., Ishimaru, Y., Ohashi, T., \& Renzini, A. 1997, ApJ, in press

Arimoto, N., \& Yoshii, Y. 1987, A\&A, 173, 23

Awaki, H., Mushotzky, R., Tsuru, T., Fabian, A. C., Fukazawa, Y., Loewenstein, M., Makishima, K., Matsumoto, H., Matsushita, K., Mihara, T., Ohashi, T., Ricker, G. R., Serlemitsos, P. J., Tsusaka, Y., \& Yamazaki, T. 1994, PASJ, 46, L65

Böhringer, H., \& Hensler, G. 1989, A\&A, 215, 147

David, L. P., Forman, W., \& Jones, C. 1991, ApJ, 380, 39

Davis, S. D., \& White, R. E., III. 1996, ApJ, 470, L35

Fujita, Y., Fukumoto, J., \& Okoshi, K. 1996, ApJ, 470, 762 (Paper I)

Fukumoto, J., \& Ikeuchi, S. 1996, PASJ, 48, 1

Hattori, M., \& Habe, A. 1990, MNRAS, 242, 399 
Hattori, M., Yoshida, T., \& Habe, A. 1995, MNRAS, 275, 1195

Irwin, J. A., \& Sarazin, C. L. 1996, ApJ, 471, 683

Jun, B. -I., Jones, T. W., \& Norman, M. L. 1996, ApJ, 468, L59

Jun, B. -I., \& Norman, M. L. 1996, ApJ, 465, 800

Kaastra, J.S. 1992, An X-Ray Spectral Code for Optically Thin Plasmas (Internal SRON-Leiden Report, updated version 2.0)

Kim, D. -W., \& Fabbiano, G. 1995, ApJ, 441, 182

Köppen, J., \& Arimoto, N. 1991, A\&AS, 87, 109

Larson, R. B. 1974, MNRAS, 166, 585

Loewenstein, M., \& Mathews, W. G. 1987, ApJ, 319, 614

Loewenstein, M., \& Mathews, W. G. 1991, ApJ, 373, 445

Loewenstein, M., Mushotzky, R. F., Tamura, T., Ikebe, Y., Makishima, K., Matsushita, K., Awaki, H., \& Serlemitos P. J. 1994, ApJ, 436, L75

Matsushita, K., Makishima, K., Awaki, H., Canizares, C. R., Fabian, A. C., Fukazawa, Y., Loewenstein, M., Matsumoto, H., Mihara, T., Mushotzky, R. F., Ohashi, T., Ricker, G. R., Serlemitsos, P. J., Tsuru, T., Tsusaka, Y., \& Yamazaki, T. 1994, ApJ, 436, L41

Matsushita, K. 1997, Ph.D. Thesis, Univ. of Tokyo

Matsumoto, H., Koyama, K., Awaki, H., Tsuru, T., Loewenstein, M., \& Matsushita, K. 1996, ApJ, in press

Mathews, W. G. 1990, ApJ, 354, 468

Matteucci, F., \& Gibson, B. K. 1995, A\&A, 304, 11

Matteucci, F., \& Tornambé, A. 1987, A\&A, 185, 51

Mewe, R., Gronenschild, E.H.B.M., \& van den Oord, G.H.J. 1985, A\&AS, 62, 197

Mewe, R., Lemen, J.R., \& van den Oord, G.H.J. 1986, A\&AS, 65, 511

Mihara, K., \& Takahara, F. 1994, PASJ, 46, 447

Mushotzky, R. F., Loewenstein, M., Awaki, H., Makishima, K., Matsushita, K., \& Matsumoto, H. 1994, ApJ, 436, L79

Nulsen, P. E. J., 1986, MNRAS, 221, 377

Rangarajan, F. V. N., White, D. A., Ebeling, W. H., \& Fabian, A. C. 1995, MNRAS, 277, 1047

Renzini, A., Ciotti, L., D'Ercole, A., \& Pellegrini, S. 1993, ApJ, 419, 52

Sarazin, C. L., \& White. R. E., III. 1987, ApJ, 320, 32 
Tammann, G. 1982, in Supernovae: A Survey of Current Research, ed. M. Rees \& R. Stoneham (Dordrecht: Reidel), 371

Note added in proof

A recent $A S C A$ result shows that the metal abundances in central regions of elliptical galaxies are $\sim 1 \mathrm{Z}_{\odot}($ Matsushita 1997).

This preprint was prepared with the AAS $\mathrm{LAT}_{\mathrm{E}} \mathrm{X}$ macros v4.0. 


\section{Figure Captions}

Fig. 1.- The schematic figure of a supernova remnant.

Fig. 2. - The evolutions of zones for (a) model A1 (solid line) and A2 (dotted line) (b) model A3 (solid line) and A5 (dotted line).

Fig. 3. - The present distribution of density. (a) A1 - A3, (b) A4 - A6, and (c) B4 - B6.

Fig. 4.- The present distribution of temperature. (a) A1 - A3, (b) A4 - A6, and (c) B4 - B6.

Fig. 5.- The present distribution of abundance $\left(Z_{m}\right)$. (a) A1 - A3, (b) A4 - A6, and (c) B4 - B6. The long-dashed line shows the metal abundance of the mass-loss gas when $Z_{\mathrm{ML}}\left(R_{e}\right)=1.0$.

Fig. 6. - The present distribution of abundance $\left(Z_{s p}\right)$ for models A3 - A6. The long-dashed line shows the metal abundance of the mass-loss gas when $Z_{\mathrm{ML}}\left(R_{e}\right)=1.0$.

Fig. 7.- Simulated X-ray spectra observed with the $A S C A$ SIS for model A4. The line shows the best fitting (two Meka plasma + absorption column density). 
Table 1. Properties of the Model Galaxies

\begin{tabular}{cccccccccc}
\hline \hline Model & $\begin{array}{c}R_{a \star} \\
(\mathrm{kpc})\end{array}$ & $\begin{array}{c}\rho_{0 \star} \\
\left(10^{-21} \mathrm{~g} \mathrm{~cm}^{-3}\right)\end{array}$ & $\begin{array}{c}R_{a h} \\
(\mathrm{kpc})\end{array}$ & $\begin{array}{c}\rho_{0 h} \\
\left(10^{-23} \mathrm{~g} \mathrm{~cm}^{-3}\right)\end{array}$ & $\begin{array}{c}R_{e} \\
(\mathrm{kpc})\end{array}$ & $\begin{array}{c}R_{t} \\
(\mathrm{kpc})\end{array}$ & $\begin{array}{c}\text { SN rate } \\
(\mathrm{SNu})\end{array}$ & $\begin{array}{c}Z_{\mathrm{ML}}\left(R_{e}\right) \\
\left(Z_{\odot}\right)\end{array}$ & $\begin{array}{c}Z_{0}\left(R_{e}\right) \\
\left(Z_{\odot}\right)\end{array}$ \\
\hline $\mathrm{A} 1$ & 0.50 & 7.20 & 5.0 & 1.75 & 6.0 & 100.0 & 0.01 & 1.0 & 1.0 \\
$\mathrm{~A} 2$ & 0.50 & 7.20 & 5.0 & 1.75 & 6.0 & 100.0 & 0.2 & 1.0 & 1.0 \\
$\mathrm{~A} 3 / \mathrm{A} 5$ & 0.50 & 7.20 & 5.0 & 1.75 & 6.0 & 100.0 & 0.01 & $0.2,1.8$ & 1.0 \\
$\mathrm{~A} 4$ & 0.50 & 7.20 & 5.0 & 1.75 & 6.0 & 100.0 & 0.01 & $0.2,1.8$ & 4.0 \\
$\mathrm{~A} 6$ & 0.50 & 7.20 & 5.0 & 1.75 & 6.0 & 100.0 & 0 & $0.2,1.8$ & 1.0 \\
$\mathrm{~B} 4$ & 0.25 & 21.8 & 2.5 & 5.30 & 3.0 & 50.0 & 0.01 & $0.2,1.8$ & 4.0 \\
$\mathrm{~B} 5$ & 0.25 & 21.8 & 2.5 & 5.30 & 3.0 & 50.0 & 0.01 & $0.2,1.8$ & 1.0 \\
$\mathrm{~B} 6$ & 0.25 & 21.8 & 2.5 & 5.30 & 3.0 & 50.0 & 0 & $0.2,1.8$ & 1.0 \\
\hline
\end{tabular}


Table 2. Properties of the Central Region

\begin{tabular}{cccccccc}
\hline \hline Model & $\begin{array}{c}n \\
\left(\mathrm{~cm}^{-3}\right)\end{array}$ & $\begin{array}{c}k T \\
(\mathrm{keV})\end{array}$ & $\begin{array}{c}Z_{m} \\
\left(Z_{\odot}\right)\end{array}$ & Model & $\begin{array}{c}n \\
\left(\mathrm{~cm}^{-3}\right)\end{array}$ & $\begin{array}{c}k T \\
(\mathrm{keV})\end{array}$ & $\begin{array}{c}Z_{m} \\
\left(Z_{\odot}\right)\end{array}$ \\
\hline A1 & 0.2 & 0.8 & 2 & B4 & 0.4 & 1.1 & 1 \\
A2 & $\ldots$ & $\ldots$ & $\ldots$ & B5 & 10 & 1.2 & 1 \\
A3 & 0.3 & 0.8 & 1 & B6 & 2 & 0.8 & 0.1 \\
A4 & 0.4 & 0.9 & 1 & & & & \\
A5 & 10 & 1.0 & 1 & & & & \\
A6 & 0.7 & 0.6 & 0.1 & & & & \\
\hline
\end{tabular}




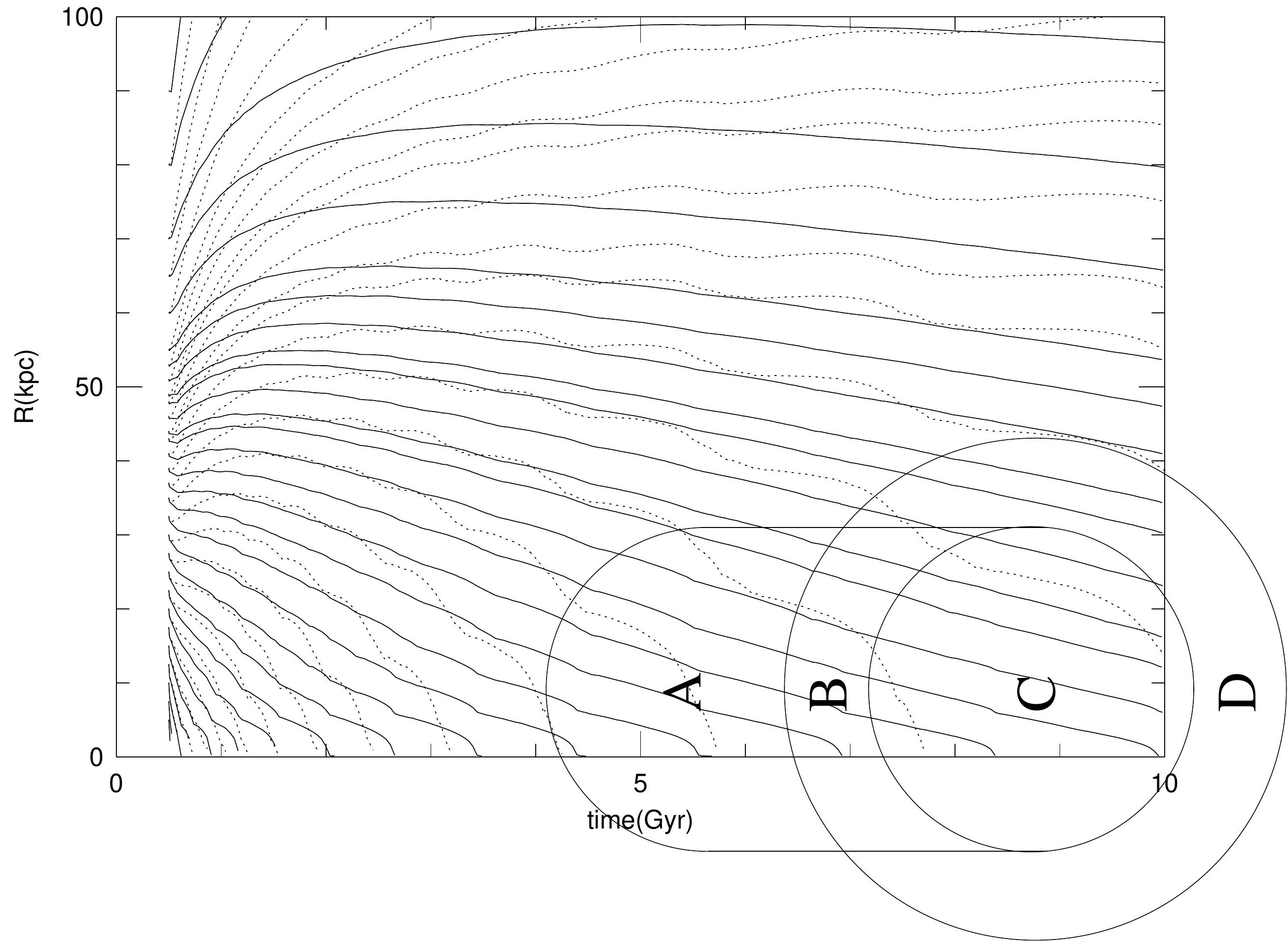




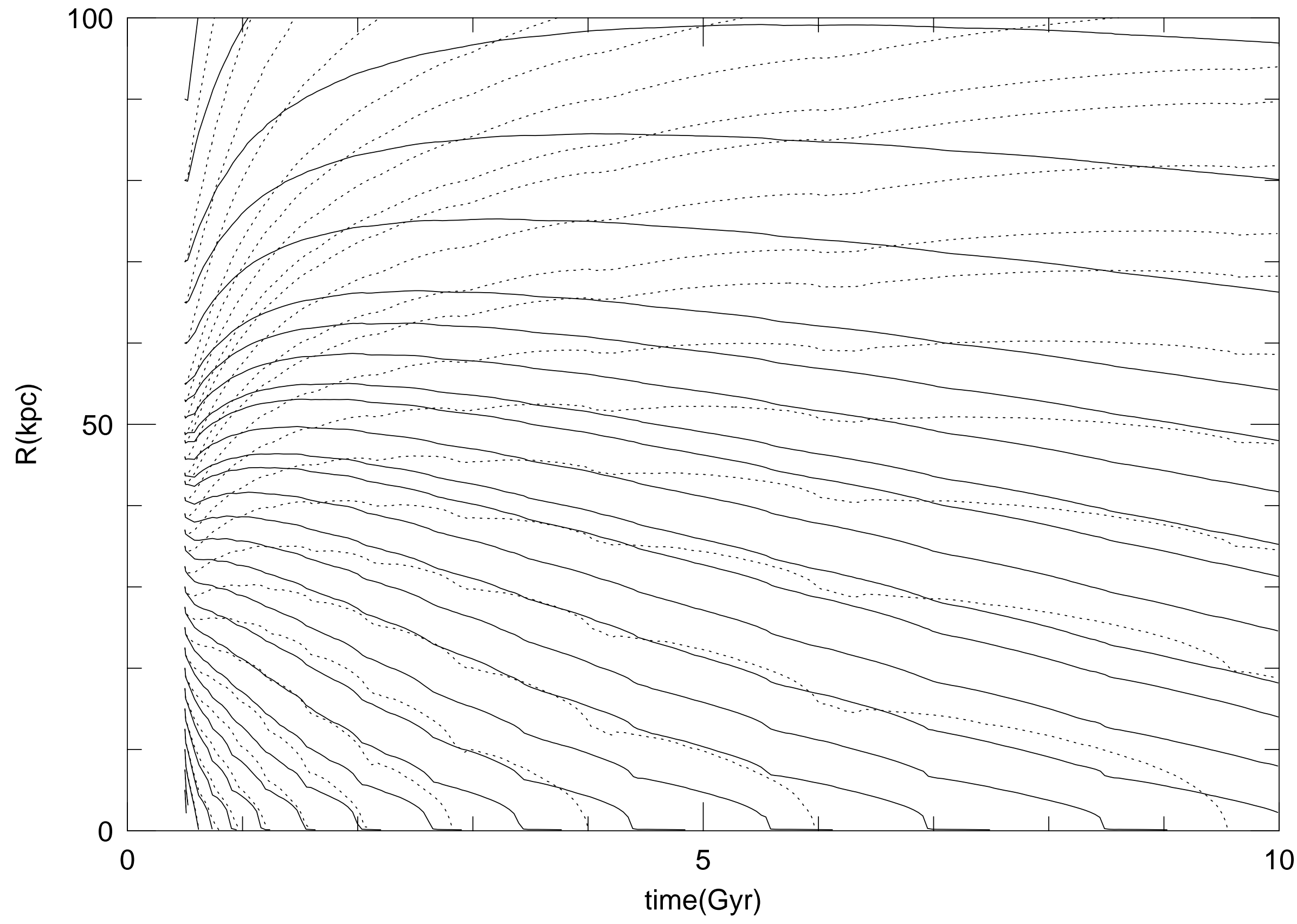




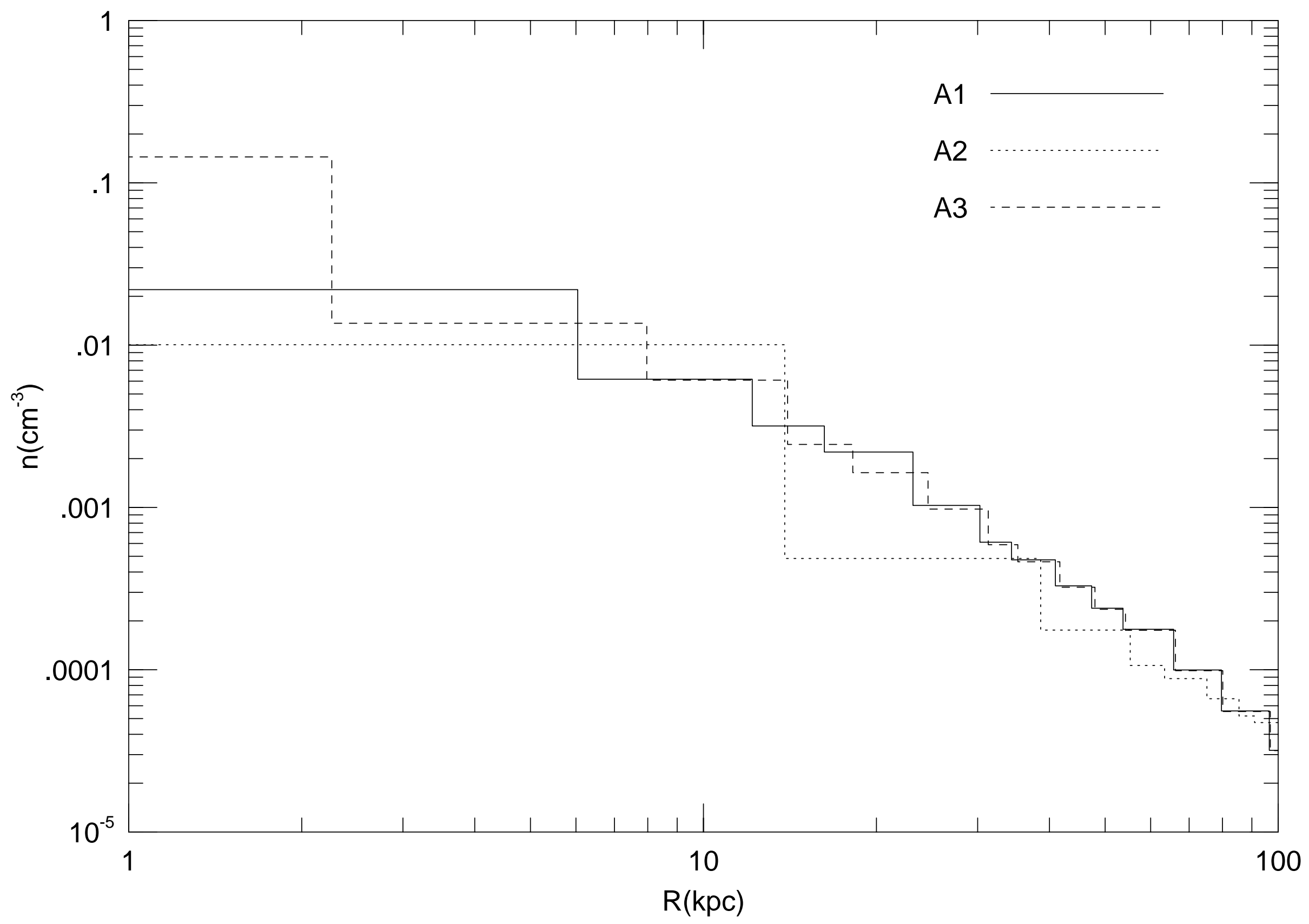




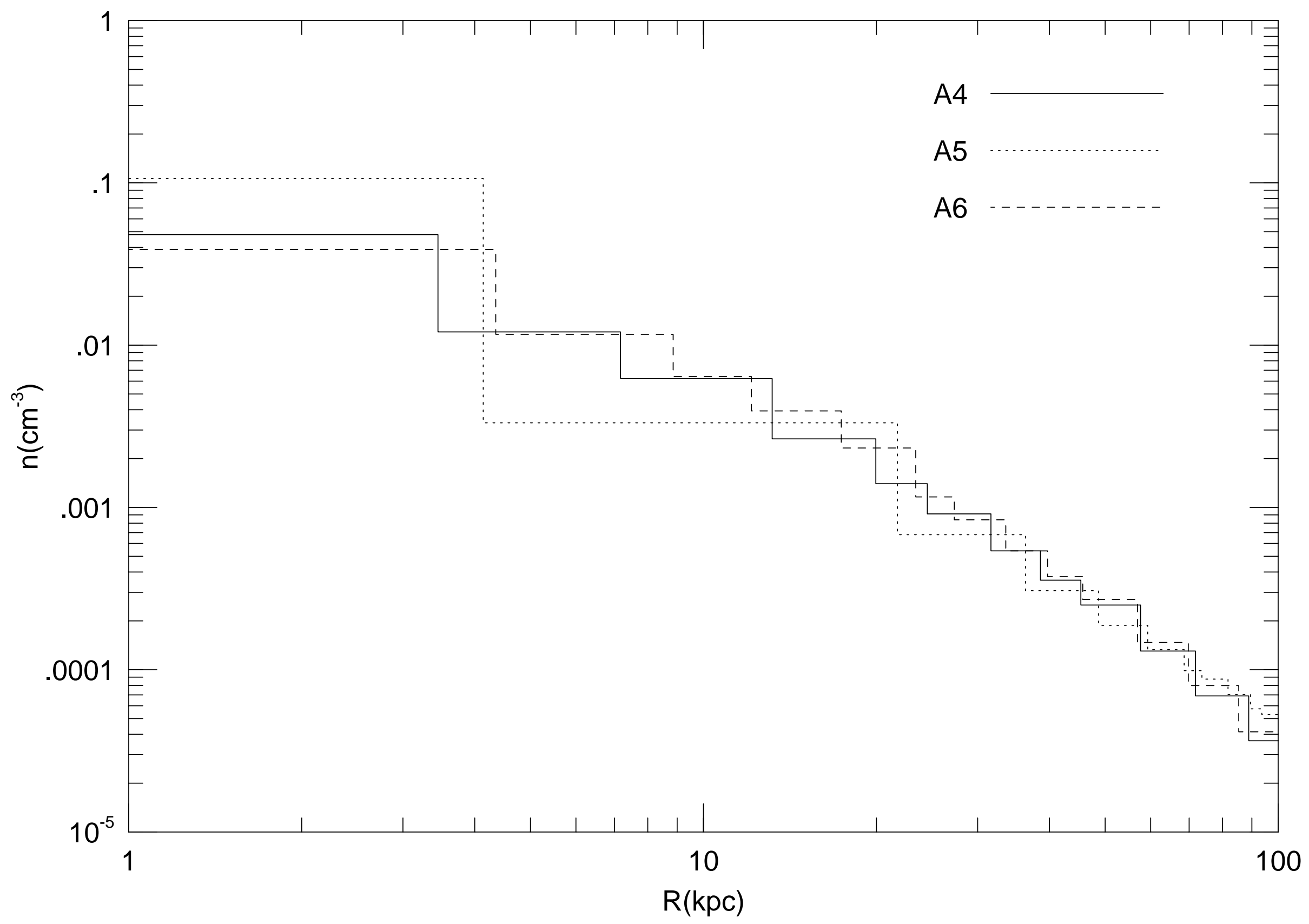




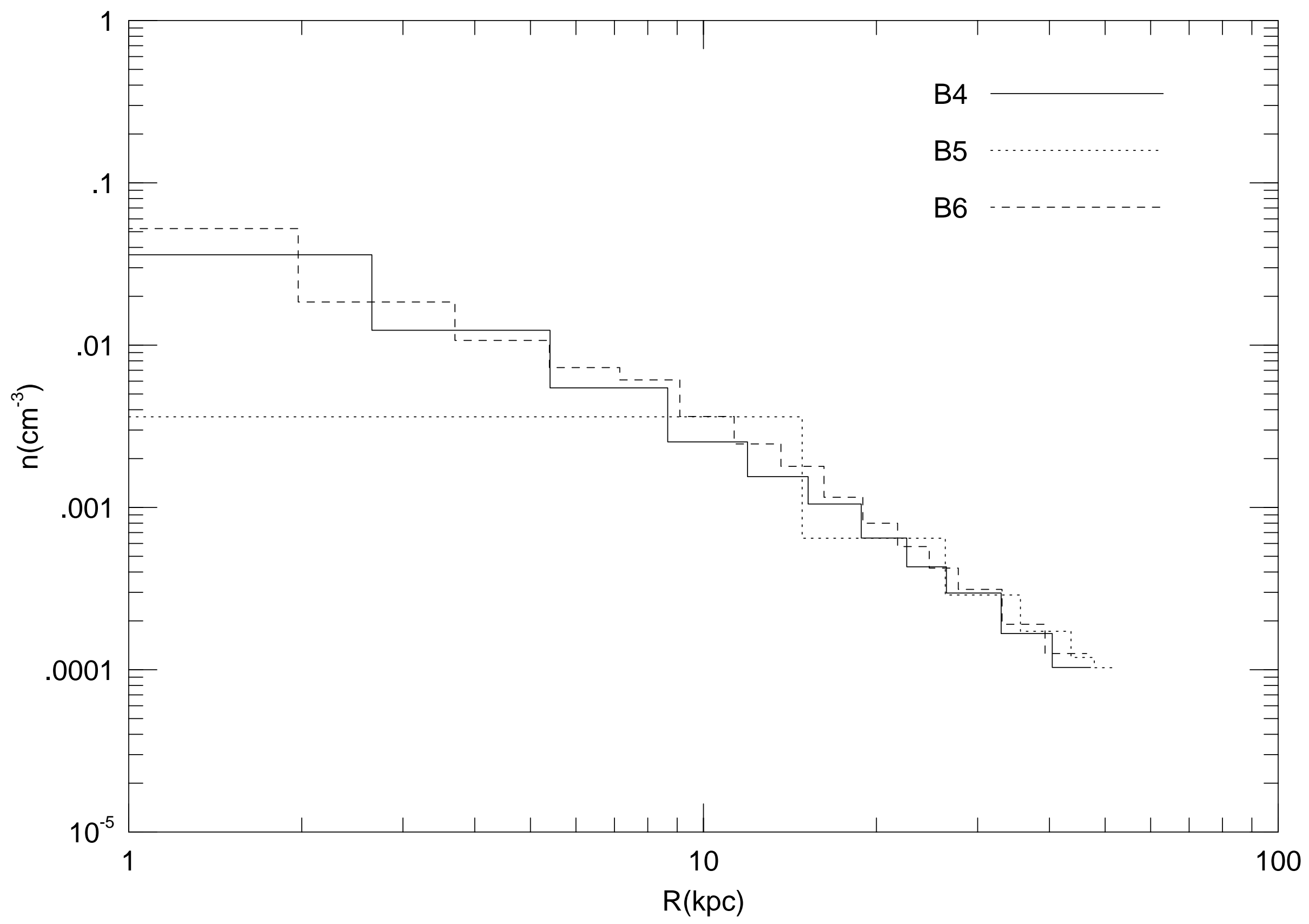




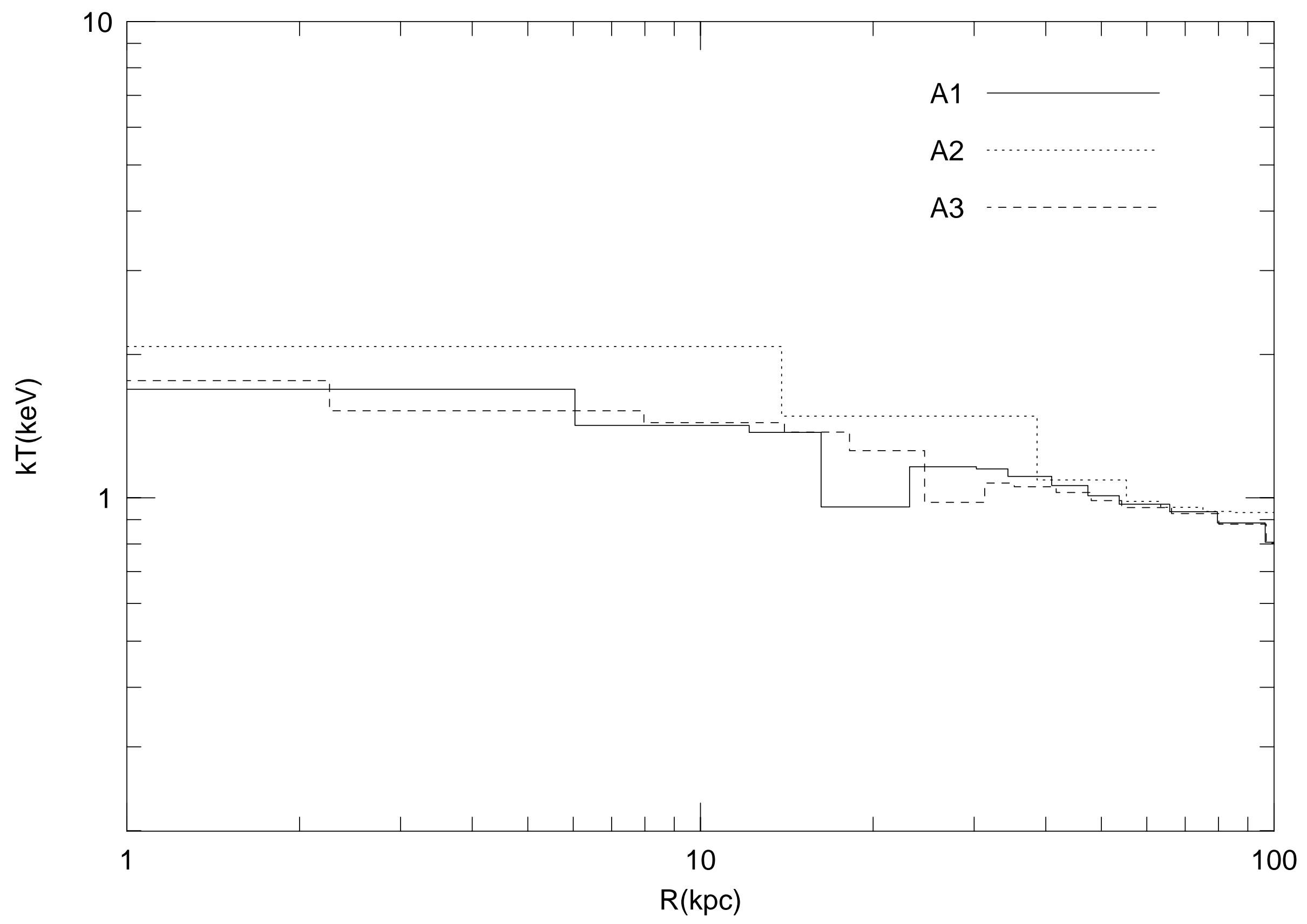




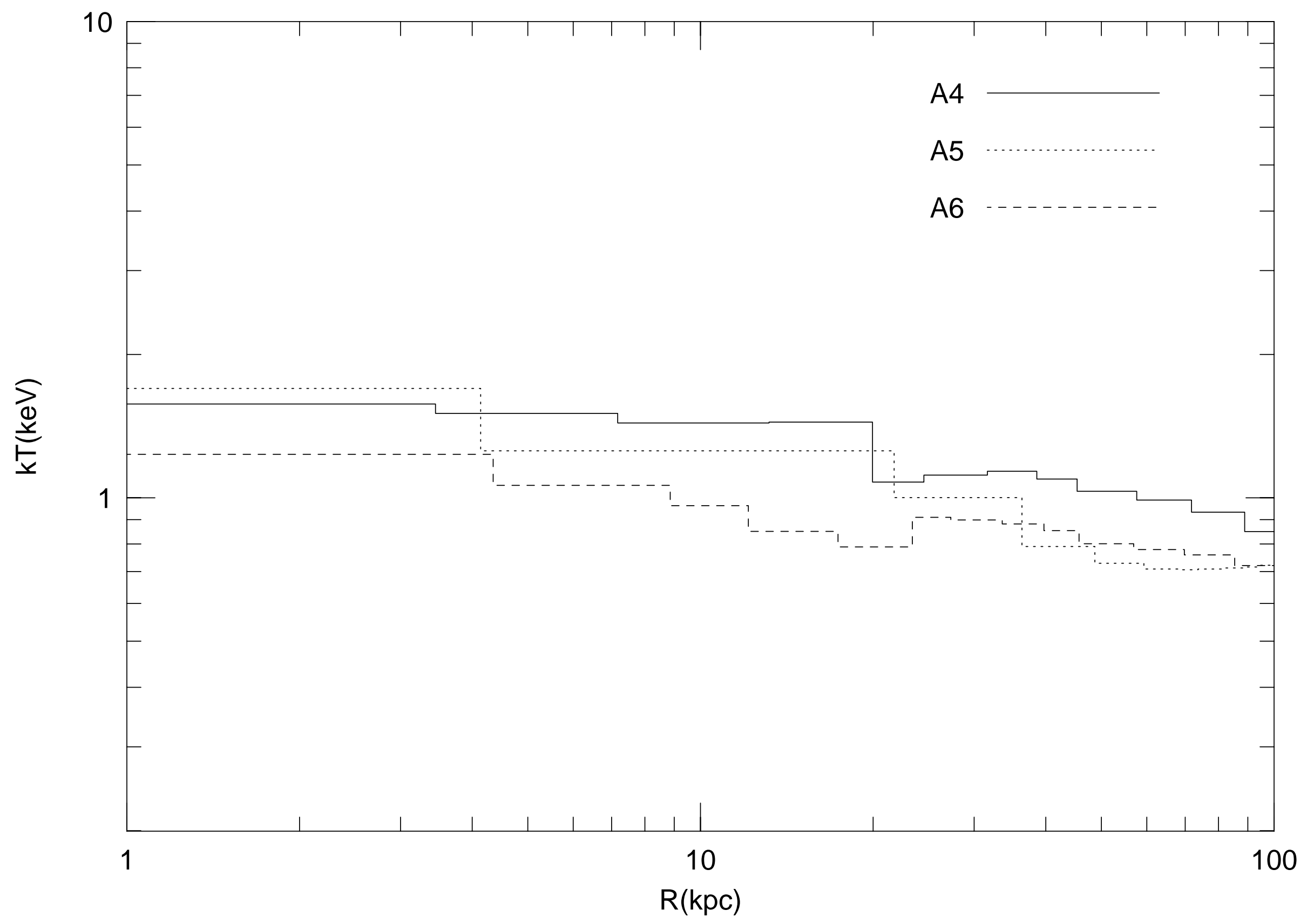




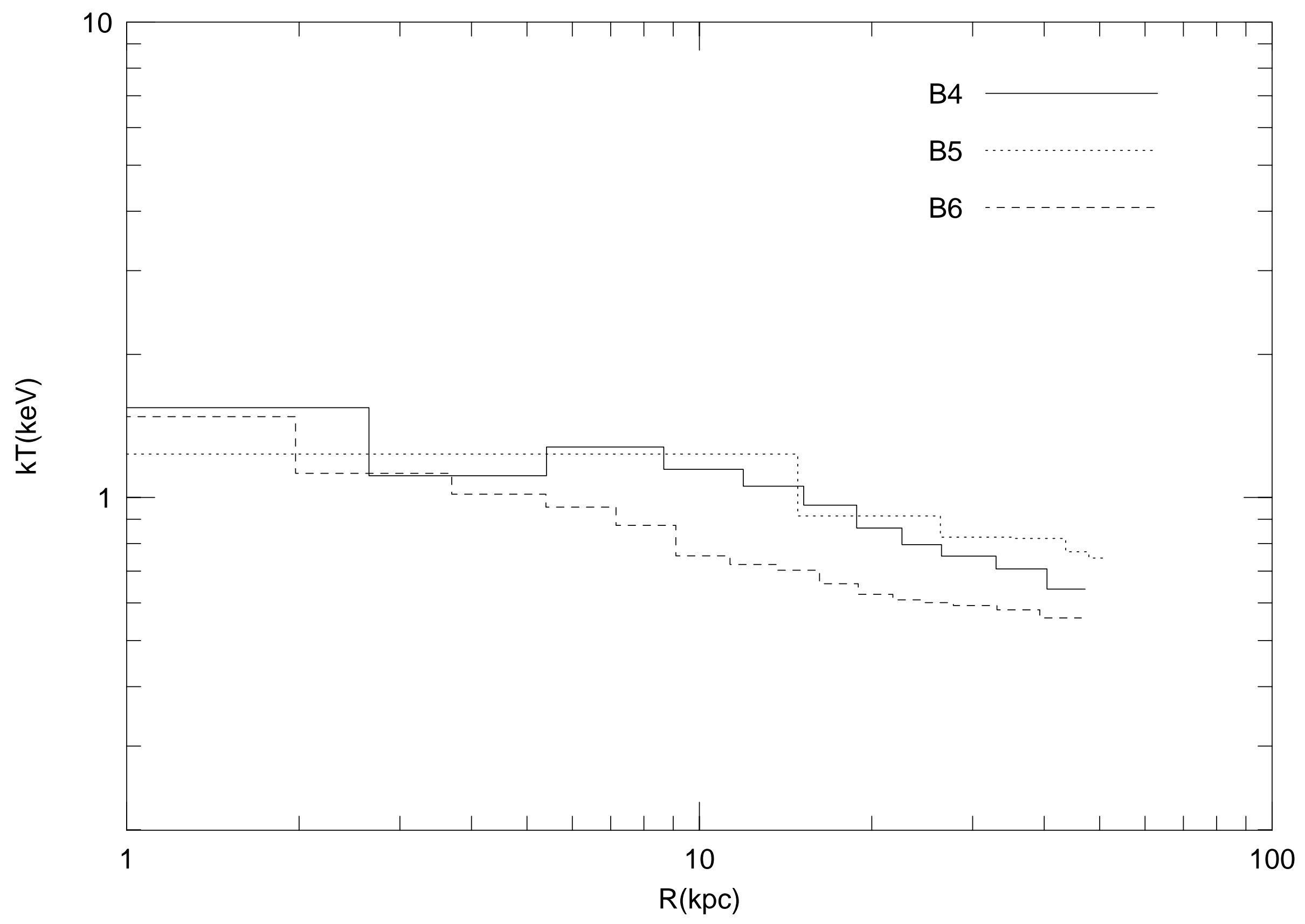




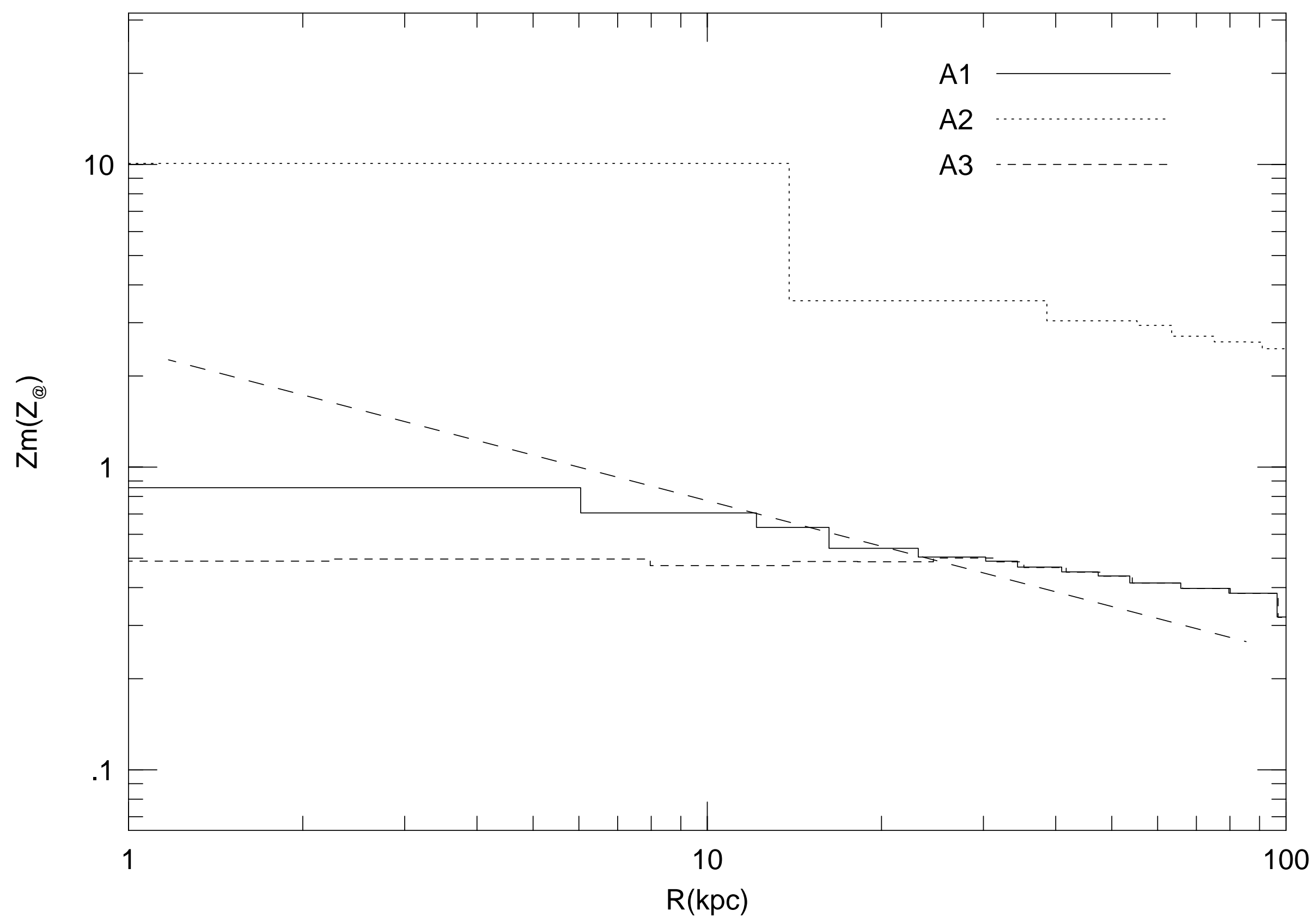




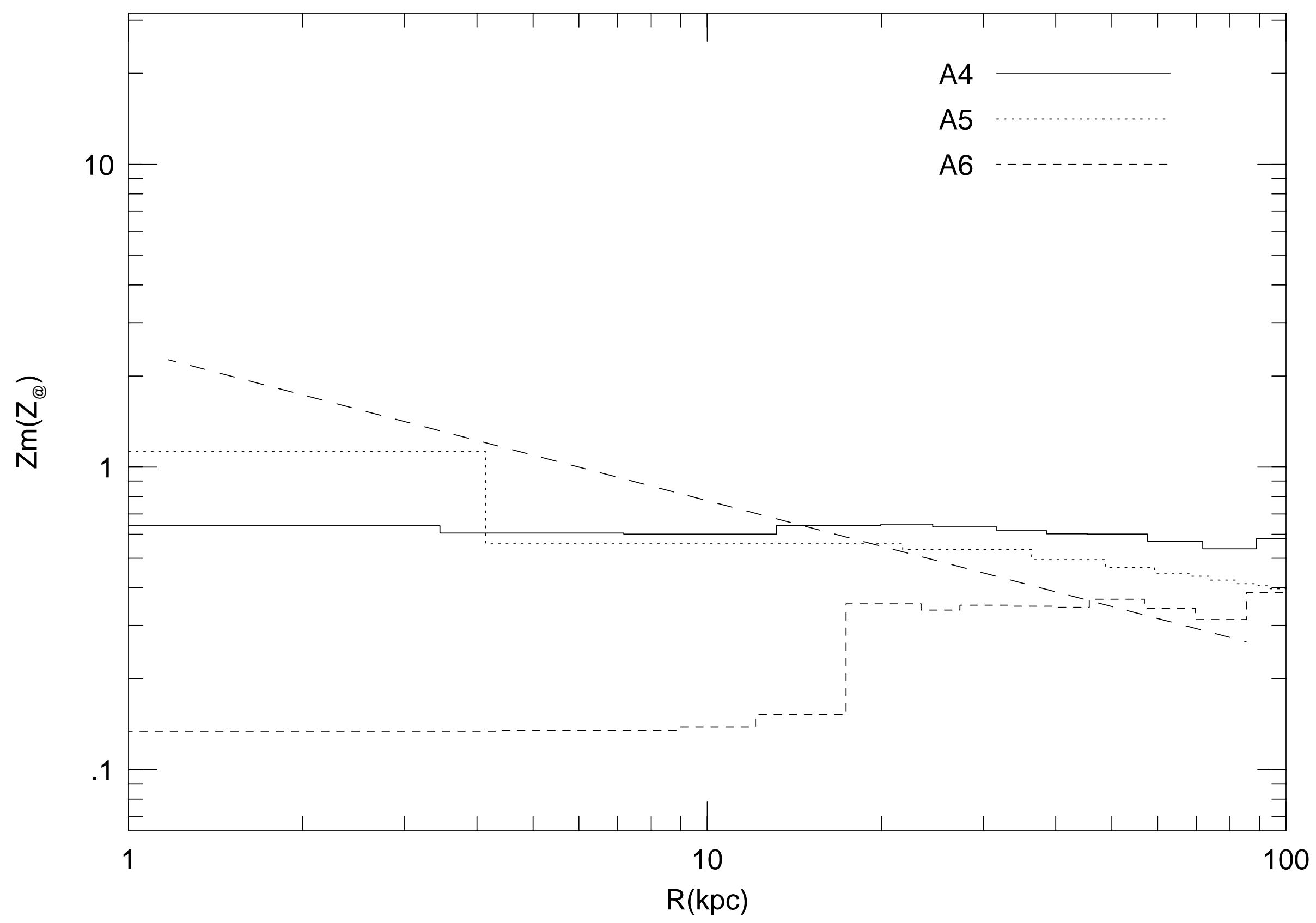




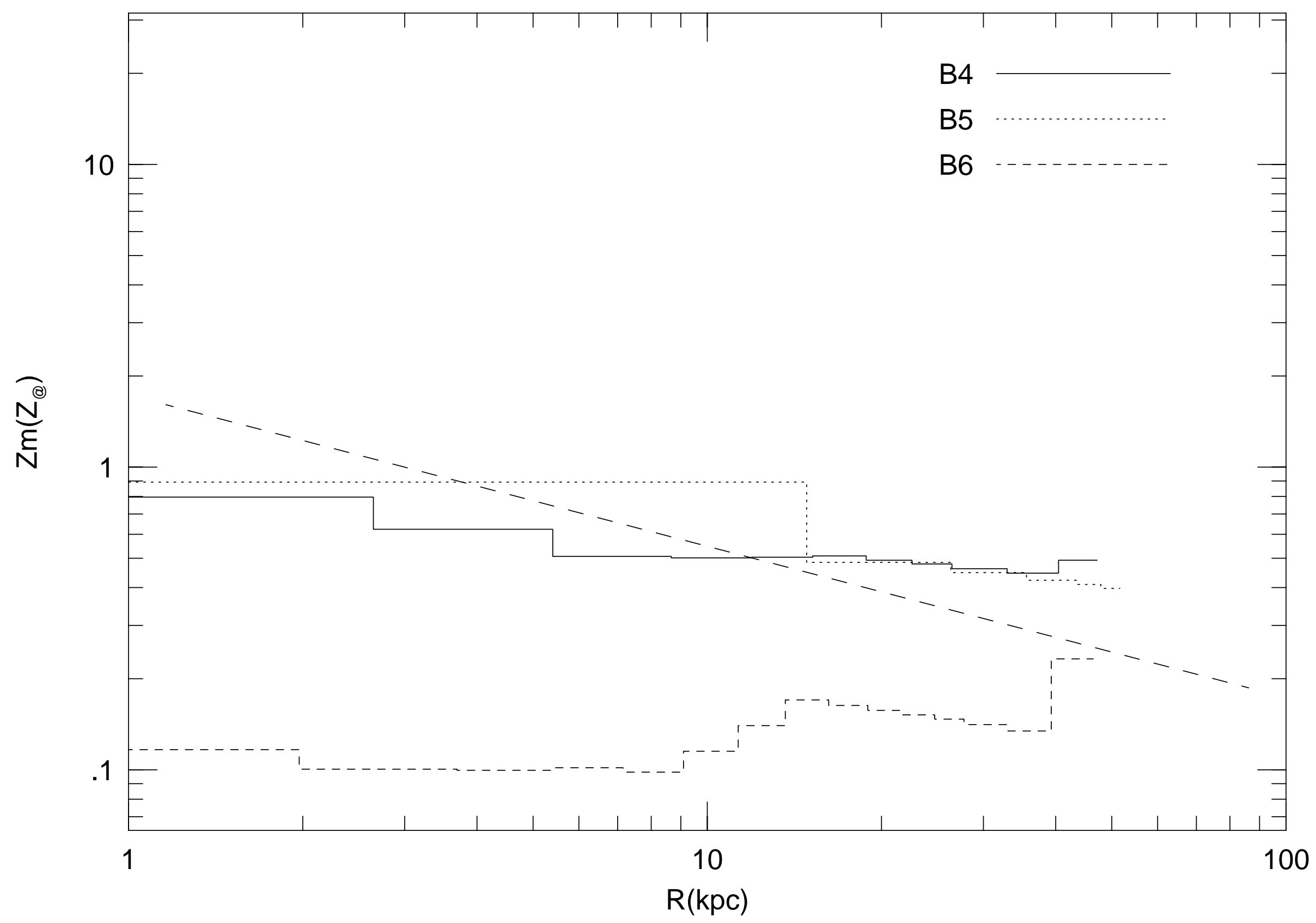




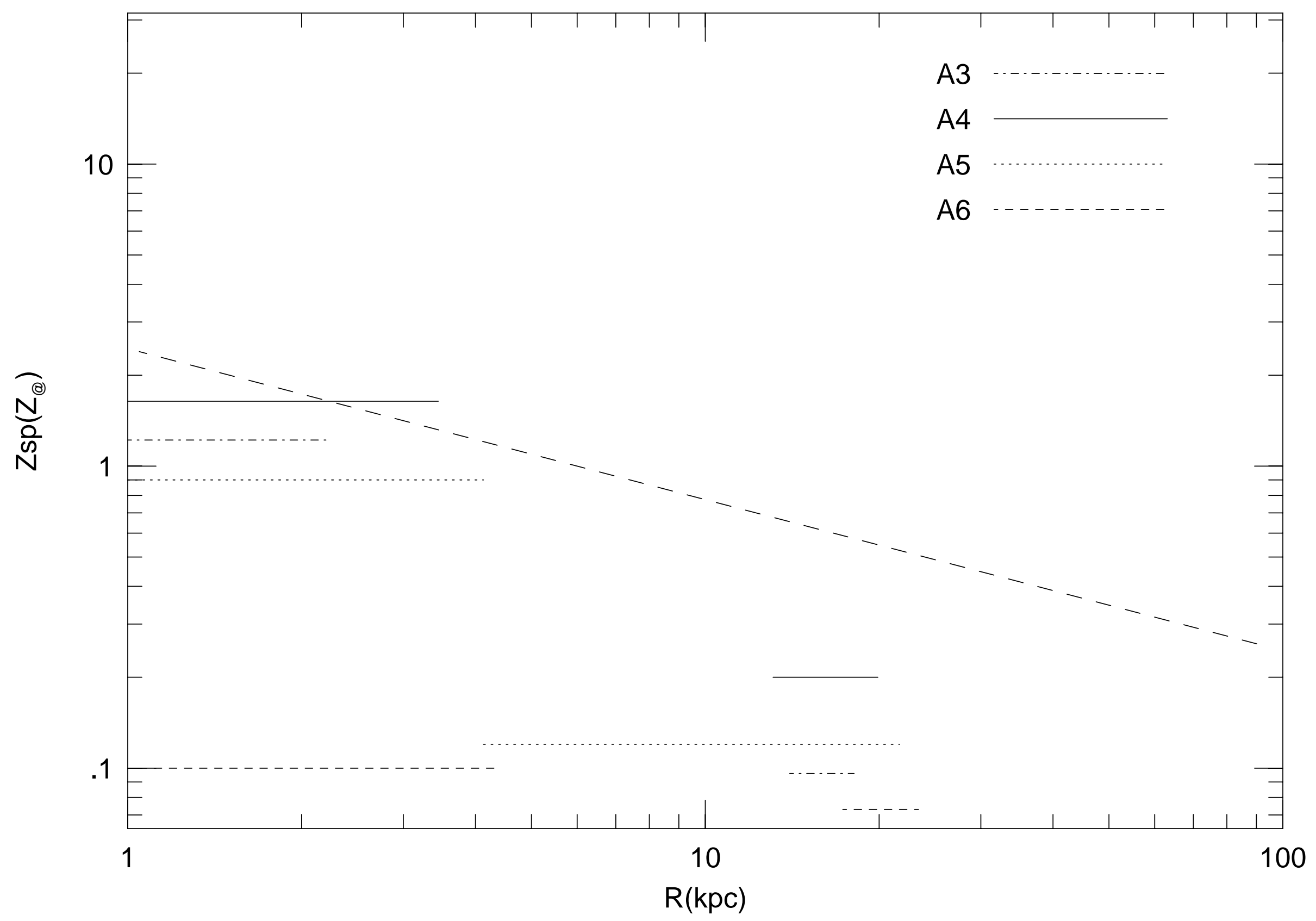




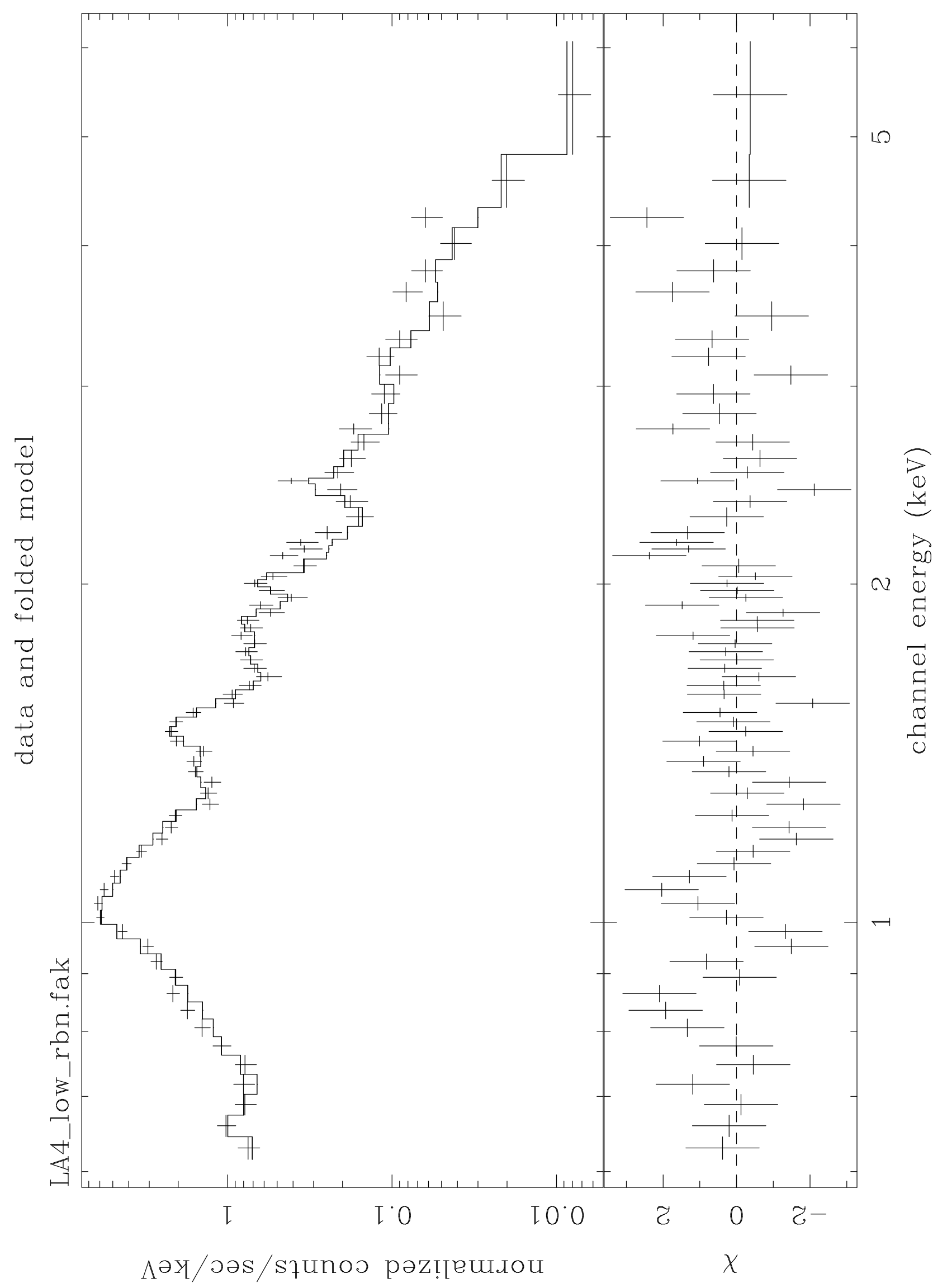

